\title{
Carbon flux on coral reefs: effects of large shifts in community structure
}

\author{
Craig Johnson ${ }^{1, *}$, David Klumpp ${ }^{2}$, John Field ${ }^{3}$, Roger Bradbury ${ }^{4}$ \\ ${ }^{1}$ Zoology Department, University of Queensland, Queensland 4072, Australia \\ ${ }^{2}$ Australian Institute of Marine Science, Townsville MC, Queensland 4810, Australia \\ ${ }^{3}$ Marine Biology Institute, University of Cape Town, Rondebosch 7700, South Africa \\ ${ }^{4}$ National Resource Information Centre, PO Box E11, Queen Victoria Terrace, Canberra, ACT 2600, Australia
}

\begin{abstract}
The effect of replacement of live coral cover by epilithic algae on patterns and magnitudes of carbon flux is examined for the shallow front slope of a midshelf reef in the Great Barrier Reef (GBR) complex of Australia. A steady-state network of carbon exchange among 19 trophic compartments is constructed for the coral-dominated state. From this, 2 scenarios for patterns of carbon flux when algae dominate are derived, viz. (1) the increase in algal production is channeled to detrital pathways (grazers do not respond), and (2) grazers utilise the increase in production of algal carbon so that transfers to detritus and grazers are in the same proportion as occurs when coral cover is high. The 3 models summarise current knowledge of carbon flux on GBR reef fronts and are compared using network analysis. Because fluxes in the reef front zone are dominated by exogenous imports and exports as a result of the high volume of water passing around and over the reef, the analyses ignore advective fluxes across the zone that are not internalised. The shift in structure to an algae-dominated system realises lower rates of benthic primary production, and thus system size and activity (i.e. total system throughput, internal throughput, development capacity and ascendancy) are reduced, suggesting a disturbed system. With loss of coral cover, the proportion of the total flow that is recycled and transferred to the detritus pool increases (although the structure of recycling is not affected), and the balance of pathways in the network is changed: average path length increases, while the average trophic level of most of the second order consumers, and trophic efficiencies of most trophic categories, decreases. Also, there are marked changes in dependencies of particular trophic groups on others. The analysis shows that, in the coral-dominated state, carbon fixed by zooxanthellae is used indirectly by most organisms in the system, even those seemingly remotely connected. Differences between the coral-and algae-dominated systems were much greater than differences between the 2 scenarios for the algae-dominated state. However, the exact fate of additional algae-derived carbon in the system is an important consideration since the 2 scenarios for the algae-dominated state yielded dissimilar values for some parameters (e.g. flow diversity, trophic dependencies and effective trophic levels of some compartments, relative importance of recycling, trophic efficiency of some trophic categories)
\end{abstract}

KEY WORDS: Carbon flux - Coral reef - Coral dominated A Algae dominated - Crown-of-thorns starfish - Network analysis

\section{INTRODUCTION}

On a global scale, many natural communities are experiencing large and fundamental changes in structure, often as a result of anthropogenic activities and often manifesting themselves as a decrease in diversity. However, whereas causality can sometimes be identified, there is little understanding of the consequences of large shifts in community structure on the processes and functioning of communities and ecosystems, de-

•E-mail: c.johnson@mailbox.uq.oz.au spite recent interest in this area (Chapin et al. 1992, Done et al, in press). Coral reefs provide a pertinent (and disturbing) marine example in that worldwide there is an increasing number of reefs that are shifting from systems dominated by coral to systems dominated by fleshy and/or turf-forming macroalgae (Wilkinson 1993, Hughes 1994). The transition to an algae-dominated community is often regarded as degradative and may be mediated by outbreaks of crown-of-thorns starfish, bleaching events, pollution, increased sedimentation, removal of grazers from the system (e.g. by overfishing), cyclones, or combinations of any of these 
(e.g. Smith et al. 1981, Moran 1986, Brown 1987, Hughes 1989, 1994, Birkeland \& Lucas 1990, Knowlton et al. 1990, Done 1992a, b, c, Glynn \& Colgan 1992, Moran et al. 1992, Glynn 1993, Sebens 1994)

Although the consequences of the shift away from reef-building corals to free-living algae are poorly understood, it is clear that changes in state to the lower diversity, algae-dominated configuration may be stable over an extended period (Hughes 1994; see also Hatcher et al. 1989, Knowlton 1992 for comment on stability of state changes) and that the transition represents a major shift in the balance of primary production from domination by zooxanthellae with a significant input from turf algae, to domination by turf algae, calcareous algae, and in many cases fleshy and foliose macroalgae. The question we address here is whether this shift significantly affects patterns of carbon flux and/or production of heterotrophic species, either as a result of changes in overall rates of primary production or in the relative magnitude of flows between different trophic groups.

State transitions to algae-dominated communities have been widespread in the central sector of the Great Barrier Reef (GBR) in Australia since the 1960s as a result of outbreaks of crown-of-thorns starfish Acanthaster planci, but not all reefs have been affected adversely (Johnson 1992, Moran et al 1992). Davies Reef, a typical midshelf platform reef in the central sector and one of the most intensively studied reefs worldwide, suffered minimal damage from starfish until the 1990s when it was moderately affected. Here we construct a steady-state model of carbon flux on the shallow windward slope of a coral-dominated reef based largely on empirical measurements from Davies Reef but augmented with data from other reef systems. From this, we derive 2 scenarios for carbon flux in the algae-dominated state which are also based on empirical measurements of primary production. Using network analysis (Wulff et al. 1989) to make quantitative comparisons among the different models, we address the question of how changes in community structure from coral- to algae-dominated systems affect network properties and patterns of carbon flux.

\section{METHODS}

The overall approach was first to assemble a steadystate network of carbon flux on a coral-dominated reef not affected by crown-of-thorns starfish, capturing as much detail in trophic structure as possible from empirical measurements. From this, 2 scenarios of carbon flow for an algae-dominated state were derived and all 3 models were then compared using network analysis. The models do not consider transitional states in the phase shift to the algae-dominated system.
Construction of steady-state models of carbon flux. In balancing the competing requirements of detail of trophic structure and availability of empirical measurements, a steady-state model of the shallow front slope (5 to $10 \mathrm{~m}$, average depth $7.5 \mathrm{~m}$ ) of Davies Reef $\left(18^{\circ} 50^{\prime} \mathrm{S}\right.$, $147^{\circ} 39^{\prime} \mathrm{E}$ ) was developed. Davies Reef is a midshelf platform reef typical of the central region of the GBR and has been studied intensively for over 2 decades. The model is an open system describing flux activity in this shallow zone and is steady state in the sense that net flow through compartments is zero (i.e. total inputs balance total outputs; note that this does not require equilibrial community structure within compartments). The network contained 19 trophic compartments (Fig. 1), which included 2 non-living compartments (water column and sedimentary detritus), 4 categories of primary producers [phytoplankton, zooxanthellae in corals, coralline algae (COA) and filamentous turf-forming algae], 4 sources of exogenous input into the system as a result of oceanic water flowing onto the reef (phytoplankton, zooplankton, water column bacteria, and water column protozoa and microzooplankton), and 5 categories of export out of the front slope zone (same as exogenous inputs, plus piscivorous fish). There was no compartment for fleshy or foliose macroalgae since, unlike other coral reef systems that become dominated by algae (Carpenter 1990, Littler et al. 1993, Hughes 1994), fleshy macroalgae are a rare component of mid- and outer-shelf GBR reefs, irrespective of coral cover.

In calculating exogenous inputs, and therefore the hydrodynamic regime of a platform reef, we ignored water flowing around the reef and considered only the input of inter-reef water that eventually leaves the reef front to flow over the reef into downstream zones. This volume is smaller (by an unknown magnitude) than the amount flowing parallel to the reef front which eventually moves around the reef to exit downstream without flowing over the reef (Hamner \& Hauri 1981, Hamner \& Wolanski 1988, Hamner et al. 1988). There were several reasons to ignore water flowing around the reef; first, the network analyses considered only the carbon internalised in the system and not that which simply passed through the zone, so ignoring water flowing around the reef had no effect on our results of network analyses. Second, carbon flowing along the slope and around (not over) the reef that is not internalised in this zone is not used by other downstream reef zone assemblages. Finally, there are no empirical estimates of the magnitude of this flux for midshelf GBR reefs.

For the coral-dominated state, coral cover was assumed to be $55 \%$ at 5 to $10 \mathrm{~m}$, where corals are the principal filter-feeding organisms (Daniel et al. 1985). The derivation of flow magnitudes is given in Appendix 1. Where empirical data for Davies Reef were not 


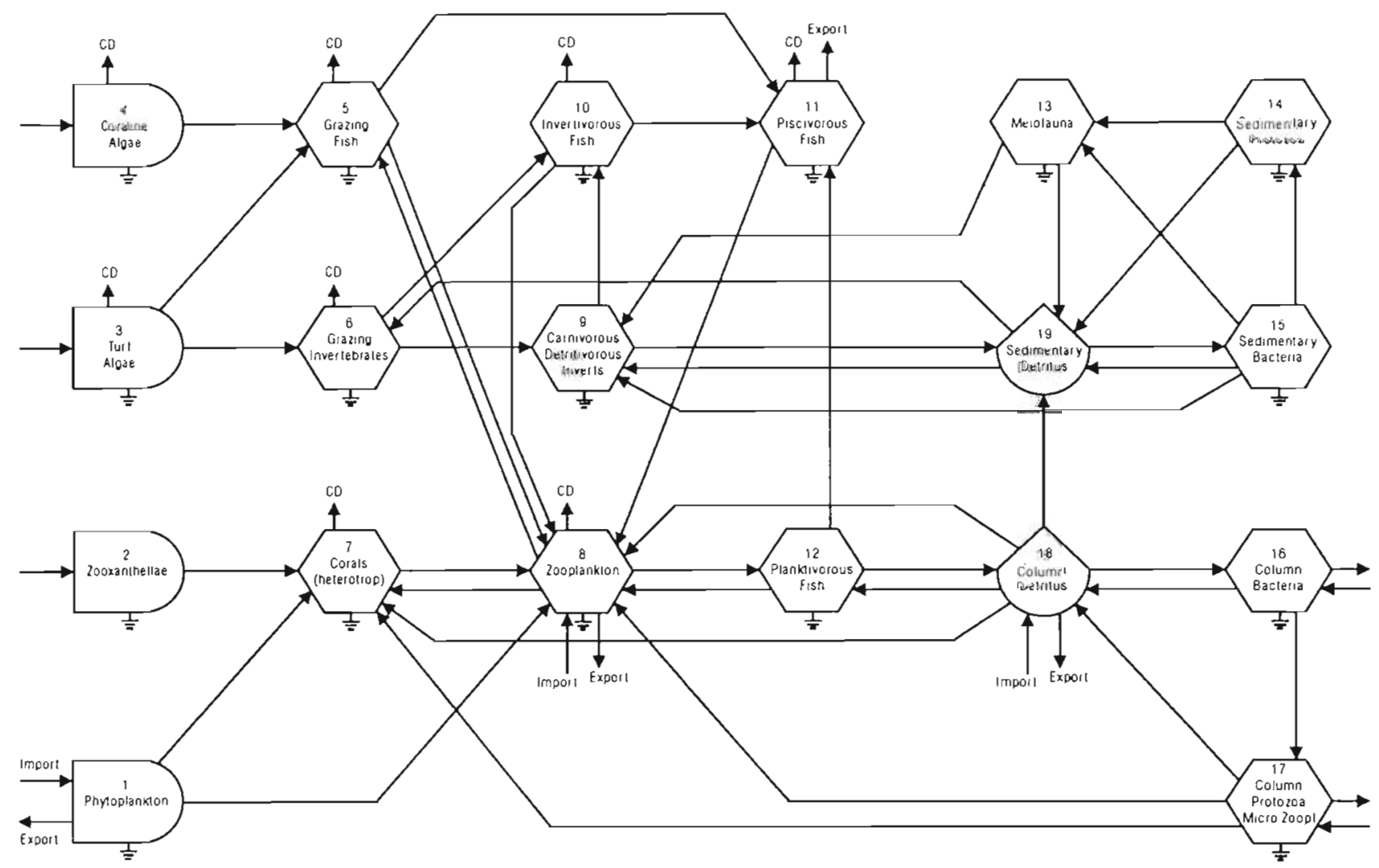

Fig. 1 Basic structure of the network representing carbon flux on the shallow front slope of Davies Reef, Australia. Flows are among 4 autotrophic, 13 heterotrophic, and 2 non-living trophic compartments. ( $\triangleq$ ) Respiration. CD: water column detritus; Corals (heterotrop): heterotrophic component of corals; Micro Zoopl: microzooplankton

available, data were used from other reefs, or flows were derived by difference assuming steady state in trophic compartments or assuming allometric behaviour of physiological parameters (Peters 1983).

The network for the coral-dominated reef was used to derive 2 models of carbon flux for the algae-dominated state (Appendix 2), viz. (1) where coral cover is reduced to $2 \%$ by crown-of-thorns starfish (Keesing 1990) and dead coral skeletons are colonised by COA and turf algae, but where grazing fish and invertebrates do not respond to the increased abundance of algae (e.g. Williams 1986, T Hart unpubl. data; see 'Discussion') and the excess in production of algal carbon is exported as detritus in the water column, and (2) where coral cover is reduced to $2 \%$ and grazers respond to the increased availability of algae (e.g. Robertson 1991) so that losses of algal carbon to grazers and losses to detritus are in the same proportion as occurs in the coral-dominated state (ca 1:1). In these models it was assumed that production of epilithic algae (COA + turf algae) per unit area of algae-covered substratum on starfish-affected reefs was identical to that on algaecovered substratum on coral-dominated reefs. This may be conservative in that preliminary data (from a single reef at a single time) suggest that algal biomass per unit area of algae-covered substratum may be up to 1.8 times higher in crown-of-thorns impacted areas (D. Klumpp unpubl. data). However, given that the area affected by starfish in Klumpp's pilot study supported a high cover of transient bluegreen algae, we favour a conservative interpretation of these preliminary measurements. Furthermore, exploratory sensitivity analyses (not presented here) for the scenario where additional algal production is lost to detritus showed that changes in gross production of autotrophs of this magnitude did not qualitatively affect comparisons. Similarly, we assumed similar productivity of turf algae for both scenarios of the algae-dominated state based on identical productivity per unit biomass of grazed (uncaged) and ungrazed turf at Davies Reef (Klumpp et al. 1987). Higher rates of algal production per unit biomass at higher standing biomass of algae appear to be a unique property of damselfish territories (Klumpp et al. 1987). On Davies Reef there is no evidence of reduced productivity of turf algae per unit biomass with reduced grazing pressure as has been reported elsewhere (see Carpenter 1990).

Despite the need for some assumptions and difficulties associated with assembling disparate measurements from a variety of sources into a system scheme, 
we contend that our values are within range for Davies Reef and preserve sensible physiological ratios and, accordingly, that our models adequately describe the flow structures in the shallow front zone of a typical midshelf platform reef in the GBR system. The appendices outline the assumptions in obtaining flux patterns; discrepancies with other systems are noted therein and in the 'Discussion'.

Network analysis (NA). A detailed comparison of the 3 models was undertaken using the NA software NETWRK3 produced by R. E. Ulanowicz (see Wulff et al. 1989). This technique allows for quantitative comparison of different network systems that have the same general structure of flows among the same kind of trophic compartments. Thus, different ecosystems of similar trophic structure (e.g. Wulff \& Ulanowicz 1989), or temporal sequences in a single system (e.g. Baird \& Ulanowicz 1989, Field et al. 1989b), can be compared. To help the reader interpret output presented here, a brief description of NA output is given below.

\section{Total system properties}

- Total System Throughput $(T S T)=\sum$ (all flows), including inputs and outputs, and is a measure of the 'size' of the system.

- Internal Throughput $(I T)=\sum$ (all flows), not including inputs (inputs include gross production of autotrophs)

- overall Finn cycling index is the proportion of TST that is cycled $=[\Sigma$ (cycled flows $)] / T S T$

- Finn cycling index for 'feeding cycles' is the proportion of total flow that is cycled in cycles where all components are living

- Detritivory $(D t)$ is the flow from detritus $\rightarrow$ trophic category II

- Herbivory $(H)$ is the flow from trophic category I $\rightarrow$ II

- relative importance of recycling $=D t / F$

- Flow Diversity $(D)$ is an information measure (on a log scale) of the 'organisation' of flows based on the number and evenness of inputs to compartments. There are several definitions of $D$ (cf. Field et al. 1989a, b. Kay et al. 1989), and here we define $D$ sensu Ulanowicz (NETWRK3 software), i.e. $D=$ A/TST, where $A=$ ascendancy

- Average Path Length (APL) is the average (or expected) number of steps (transfers) a unit of medium (in this case, carbon) experiences in passing through the network; $A P L=(T S T-E I) / E I=I T / E I$, where $E I$ is the total of exogenous inputs (and includes the gross production of autotrophs)

- Ascendancy $(A=T S T \times D)$ is an information-based measure of both size and organisation of flows. A tends to increase with increasing trophic specialisation, successional stage towards 'maturity' of a system, internalisation, and increased cycling. De- creases in $A$ are usually interpreted as indicative of stress

- Development Capacity (DC) defines the potential for a network to develop, i.e. defines the maximum possible value of $A$. DC can be viewed as a measure of the 'total uncertainty' of the network, and $A$ as the amount of uncertainty resolved by knowing the flow structure

- Overheads $(D C-A)$ define residual uncertainty in flow structure and are partitioned into components due to respiration, inputs, exports and redundancy $(R d)$, where $R d$ is the residual uncertainty associated with multiple or parallel pathways

\section{Trophic analysis and compartmental attributes}

- Dependency Coefficients are the fraction of total flow leaving one compartment that enters another compartment (including direct, indirect and recycled flows)

- the Effective Trophic Level of a compartment is a weighted average of the compartment's trophic level, where weightings are the relative amounts of total influx into the compartment at different trophic levels; by convention the trophic level of autotrophs and detritus $=1$

- Trophic Efficiency is the comparison of inflow into a trophic category with outflow available to the next trophic category (= ecological efficiency)

In addition, network analysis (1) extracts all biogeochemical cycles and identifies subgroups of cycles, referred to as nexuses, that share the same smallest, or limiting, transfer and (2) enables derivation of a Lindeman spine, which is an abstract food chain in which the network is collapsed and the system summarised by describing fluxes between successive trophic categories or levels (note that a single trophic compartment or 'trophic guild' may be represented in several trophic categories)

\section{RESULTS}

\section{Total system properties}

\section{General}

Carbon flux in the shallow (5 to $10 \mathrm{~m}$ ) front zone of Davies Reef is dominated by exogenous inputs and exports as a result of transport of particulate organic carbon (POC) in water moving onto the reef (see Compartments $1,8,16,17,18$, Appendix 1). The flow velocity and amount of POC carried generates inflows and outflows of carbon several orders of magnitude greater than individual transfers within the zone. However, since the great majority of exogenous carbon in 
the water column is swept into and out of the front reef zone without being internalised (i.e. most transport is across the reef front and then around the reef to exit downstream, while a smaller volume flows into the reef flat zone; see Hamner \& Hauri 1981, Hamner \& Wolanski 1988, Hamner et al. 1988), the network analyses were undertaken ignoring the high throughflow of exogenous carbon

\section{System size and overall flow structure}

In the transition from a coral- to an algae-dominated system, the amount of carbon fixed in benthic primary production decreases from ca 15.9 to $3.0 \mathrm{~g} \mathrm{C} \mathrm{m}^{-2} \mathrm{~d}^{-1}$ (三13.17 and $1.81 \mathrm{~g} \mathrm{C} \mathrm{m}^{-2} \mathrm{~d}^{-1}$ net primary production, NPP) since in this system live coral is more productive than an equivalent area of dead coral covered with epilithic algae (see 'Discussion'). This effects a decrease in the total system measures that are based on absolute magnitudes of flows (i.e. internal and total system throughputs, ascendancy, development capacity and overheads; see Table 1, Fig. 2, Appendices 1 \& 2). Similarly, the transfer of carbon between trophic categories $\mathrm{I} \rightarrow \mathrm{II}, \ldots, \mathrm{IV} \rightarrow \mathrm{V}$ is $\sim 4$ to 14 times lower in the algaedominated system, depending on the particular transfer (Fig. 2). Not surprisingly, overall flow magnitudes for the algae-dominated state are slightly greater when a proportion of the increased biomass of algae is consumed by grazers and passed on through the food web instead of being channeled to the detritus pool (Table 1).

The greatest diversity of flows is realised in the algae-dominated configuration where grazers respond to the increase in algal production (Table 1), which reflects a greater evenness of flows, particularly from primary producers $\rightarrow$ first order consumers $\rightarrow$ second order consumers. In the coral-dominated state, primary production and flux of plant carbon to consumers is dominated by zooxanthellae and transfer from zooxanthellae to coral tissue $(93$ and $98 \%$ respectively for the coral-dominated state, versus 18 and $40 \%$ for depleted coral cover where grazers respond to the increase in algae). The greater average path length in the algae-dominated configurations (Table 1 ) is also attributable to turf and coralline algae accounting for a greater proportion of the primary production after loss of coral cover. In the coral-dominated state, a greater amount of carbon fixed by zooxanthellae travels only 2 steps before being lost via export of zooplankton, whereas when turf and coralline algae account for most of the primary production, a unit of flux will, on average, travel more steps before exiting the system (Fig 1, Appendices $1 \& 2$; note that in balancing models in which 'exogenous' carbon is ignored, there is no export of water-column detritus in any state of the system and export of zooplankton only occurs when coral dominates).

\section{Recycling}

The structure of recycling is similar in all models and is relatively complex. All models contain 78 cycles, there are only 12 single-cycle nexuses $(15.4 \%$ of all cycles), and there are 2 large nexuses, one containing 20 cycles and one containing 16 cycles. All cycles in both large nexuses include transfers via sedimentary and/or water column detritus, and the largest cycles (up to 10 transfers in the circuit loop) also include flows through both detritus categories. Flows via detritus are also important in many of the smaller nexuses, with $88.5 \%(69)$ of all cycles involving water column detritus and $66.7 \%$ (52) involving sedimentary detritus. However, despite the importance of both forms of detritus in recycling, bacteria play a relatively minor role since water column bacteria are involved in only $16.7 \%(13)$ of cycles and sedimentary bacteria in only $35.9 \%$ (28). Moreover, the magnitude of flows via bacteria is small (Appendices 1 \& 2), and transfers involving bacteria are the critical or limiting transfer in

Table 1 Comparison of total system properties of network models of coraland algae-dominated states of the shallow reef slope of Davies Reef. Exogenous inputs not internalised in the system are not included in these analyses. ' $\uparrow$ in algal $C^{\prime}$. increase in algal carbon in the shift from a coral- to an algaedominated state. Units of $\mathrm{g} \mathrm{C} \mathrm{m}^{-2} \mathrm{~d}^{-1}$

\begin{tabular}{|c|c|c|c|}
\hline \multirow[t]{2}{*}{ Parameter } & \multirow{2}{*}{$\begin{array}{c}\text { Coral } \\
\text { dominated }\end{array}$} & \multicolumn{2}{|c|}{ Algae dominated } \\
\hline & & $\begin{array}{l}\uparrow \text { in algal } C \text { to } \\
\text { detritus }\end{array}$ & $\begin{array}{l}\uparrow \text { in algal } \mathrm{C} \text { to } \\
\text { grazers \& detritus }\end{array}$ \\
\hline Total system throughput ${ }^{*}$ & 82.97 & 26.62 & 27.38 \\
\hline Internal throughput ${ }^{*}$ & 64.64 & 21.73 & 22.32 \\
\hline Full development capacity & 233.2 & 77.0 & 81.1 \\
\hline Full ascendancy & 155.7 & 50.0 & 52.7 \\
\hline Overhead (inputs) & 4.21 & 1.86 & 1.78 \\
\hline Overhead (exports) & 0.71 & 0.11 & 0.16 \\
\hline Overhead (respiration) & 26.78 & 10.00 & 10.74 \\
\hline Redundancy & 45.84 & 15.03 & 15.70 \\
\hline Flow diversity & 1.88 & 1.88 & 1.93 \\
\hline Average path length & 3.53 & 4.44 & 4.41 \\
\hline $\begin{array}{l}\text { Finn cycling index (feeding } \\
\text { cycles only) }\end{array}$ & 0.033 & 0.0038 & 0.0038 \\
\hline Overall Finn cycling index & 0.258 & 0.324 & 0.316 \\
\hline Relative import. of recycling & 0.98 & 7.39 & 5.31 \\
\hline Detritivory ${ }^{\circ}$ & 13.47 & 7.11 & 7.11 \\
\hline Herbivory ${ }^{\circ}$ & 13.7 & 0.96 & 1.34 \\
\hline
\end{tabular}



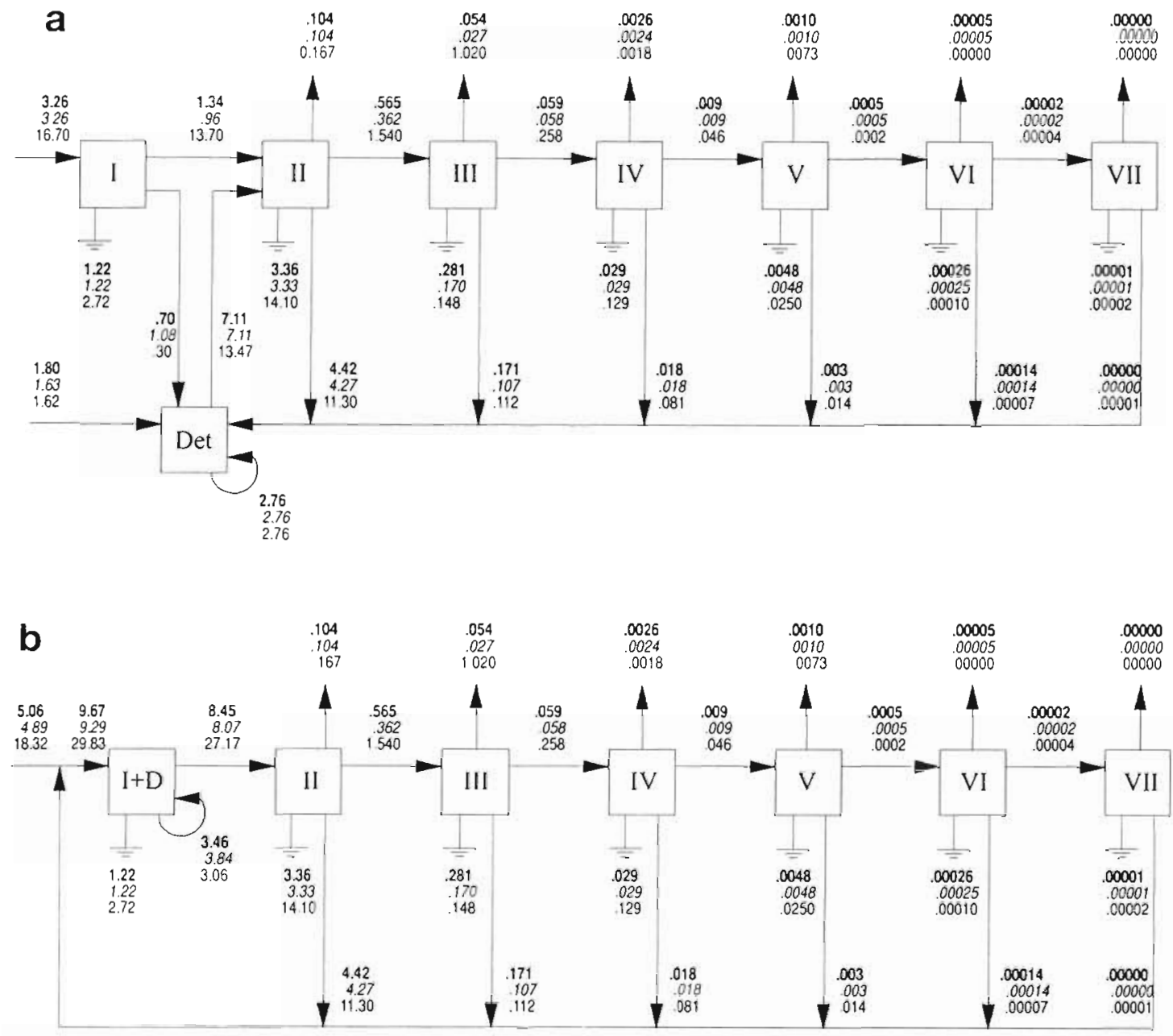

Fig. 2. Lindeman spines summarising trophic structure in which (a) detritus and autotrophs are separate compartments, and (b) where detritus and autotrophs are pooled in the same compartment. Bottom values (normal print) are for the coral-dominated state, middle values (italics) are for the algae-dominated state where the increase in algal production goes entirely to detritus (Scenario 1), and top values (bold) are for the algae-dominated state where grazers consume a proportion of the increase in algal production (Scenario 2). Member compartments of trophic categories I to VII can be determined from Fig. 1 by following network pathways and counting the number of transfers from detritus and primary producer compartments; we adopted the standard convention that primary producers and detritus are at trophic category 1

6 cycles. The only qualitative difference in overall flow structure among the models is that in the algaedominated configuration where grazers increase their consumption of algae, there is only 1 nexus of 2 cycles and 2 containing 4 cycles, whereas the other models have 3 nexuses of 2 cycles and only 1 containing 4 cycles.

The amount of total flow activity that is recycled is high in all models but varies from $25.8 \%$ in the coraldominated state to $32.4 \%$ in the algae-dominated state when the increase in algal production is transferred to detritus (overall Finn cycling index, Table 1). The overall increase in the proportion of recycled material with loss of coral cover is indicative of the reduced total system throughput but also suggests that recycling among non-coral components is greater than that among trophic groups interacting directly with corals. The relative importance of recycling ( = ratio of detritivory: herbivory) increases dramatically with loss of coral cover because of the large decrease in 'herbivory'; note that for the purposes of network analysis, corals utilising carbon fixed by zooxanthellae are classified as 'herbivores' The importance of recycling is greatest when increases in algal production following depletion of corals are channeled to detritus, since in this scenario herbivory is lowest.

In contrast to the trend in the overall cycling index. the Finn cycling index for feeding cycles is an order of magnitude lower when algae dominate (Table 1). This reflects that cycling among living trophic compartments is dominated by exchanges between corals and zooplankton when coral cover is high 


\section{Trophic analysis}

\section{Trophic structure}

The analysis identified 9 trophic categories (e.g. piscivorous fish are at trophic level IX in transfers from sedimentary detritus $\rightarrow$ sedimentary bacteria $\rightarrow$ sedimentary protozoa $\rightarrow$ meiofauna $\rightarrow$ detritivorous invertebrates $\rightarrow$ invertivorous fish $\rightarrow$ zooplankton $\rightarrow$ planktivorous fish $\rightarrow$ piscivores). However, flows associated with trophic categories VIII and IX are minimal, and are therefore not considered or included in the Lindeman spines (Fig 2)

The Lindeman spines (Fig. 2) summarise trophic structure after collapsing of the system to simple linear transfers between trophic categories. They summarise several salient differences among the models, viz. the greater 'size' of the coral-dominated system; increased flows to detritus from primary producers in the algaedominated state, particularly if grazers do not respond to the increase in algal production; and, in comparing between the 2 algae-dominated scenarios, differences in flows from plants to grazers to higher order consumers are rapidly damped (largely because of low trophic efficiency of trophic category II; Table 2) so that flows from trophic categories III $\rightarrow$ IV are similar in the 2 models, and flows to higher trophic categories are essentially identical.

Despite the large number of trophic levels, the average level of feeding of all trophic groups in all models is always $<4$ (Table 3). The effective trophic level (ETL) of grazing fish (which inadvertently feed on demersal zooplankton; Polunin 1988, Klumpp \& Polunin 1989) and the higher order consumers of zooplankton, planktivorous fish and piscivorous fish, declines with decreasing coral abundance (Table 3). This indicates that in the coral-dominated state carbon fixed by zooxanthellae is eventually utilised by higher order consumers in significant amounts and that in the transition to an algae-dominated system, the average number of steps from photosynthetically fixed carbon to consumers decreases. However, the decline in the ETL of zooplankton with loss in coral cover is, in one sense, artificial since the link from corals to zooplankton describes the reproductive output of corais and does not indicate that zooplankton feed directly on corals (although zooplankton feed indirectly on corals by consuming coral mucus in the water column; see Appendix 1; Gottfried \& Roman 1983). Transfer from corals to fish is largely via the contribution of coral gametes and larvae to zooplankton, but there are some links via production of mucus and its loss to the water column as detritus. The ETLs of compartments in trophic chains involving microbes, detritivores, and invertebrates (and therefore invertivorous fish) are largely unaffected by the transition from coral- to algae-dominated (Table 3). The exception is the ETL of water column protozoa which increased from 1.54 to 3 , but this reflects only that imports of column protozoa are not required to balance the algae-dominated networks

Trophic efficiencies of trophic categories II and III distinguish between the 2 scenarios for the algaedominated system (Table 2). Compared to the coraldominated system, the trophic efficiency of trophic category II decreases in the algae-dominated state if the increase in algal carbon is lost to detritus, but increases

Table 2. Trophic efficiencies ( $\%$ ) of each trophic category for each of the 3 models. ' $T$ algal $C$ ' increase in algal carbon in the shift from a coral- to an algae-dominated system

\begin{tabular}{|lccc|}
\hline $\begin{array}{l}\text { Trophic } \\
\text { category }\end{array}$ & $\begin{array}{c}\text { Coral } \\
\text { dominated }\end{array}$ & $\begin{array}{c}\text { Algae dominated } \\
\uparrow \text { algal C to } \\
\text { detritus }\end{array}$ & $\begin{array}{c}\uparrow \text { algal C to } \\
\text { grazers \& detritus }\end{array}$ \\
\hline II & 5.7 & 4.5 & 6.7 \\
III & 16.8 & 15.9 & 10.4 \\
IV & 18.0 & 15.6 & 15.3 \\
V & 0.5 & 5.1 & 5.2 \\
VI & 16.5 & 4.2 & 4.1 \\
VII & 0.0 & 0.0 & 0.0 \\
Average & 11.5 & 9.06 & 8.34 \\
\hline
\end{tabular}

Table 3. Effective trophic levels of each trophic compartment for each model; values for autotrophs and detrital compartments $=1$ by convention. ' $\uparrow$ algal $C$ ' increase in algal carbon in the shift from a coral- to an algae-dominated system

\begin{tabular}{|lccc|}
\hline Compartment & $\begin{array}{c}\text { Coral } \\
\text { dominated }\end{array}$ & $\begin{array}{c}\text { Algae dominated } \\
\uparrow \text { algal C to } \\
\text { detritus }\end{array}$ & $\begin{array}{c}\uparrow \text { algal C to } \\
\text { grazers \& detritus }\end{array}$ \\
Grazing fish & 2.19 & 2.06 & 2.03 \\
Grazing invertebrates & 2.00 & 2.00 & 2.00 \\
Corals (heterotrophic) & 2.00 & 2.01 & 2.01 \\
Zooplankton & 2.94 & 1.57 & 1.57 \\
Carniv/detrit inverts & 2.00 & 2.00 & 2.00 \\
Invertivorous fish & 3.00 & 3.00 & 3.00 \\
Piscivorous fish & 3.47 & 3.15 & 3.08 \\
Planktivorous fish & 3.46 & 2.43 & 2.43 \\
Meiofauna & 3.13 & 3.13 & 3.13 \\
Sedimentary protozoa & 3.00 & 3.00 & 3.00 \\
Sedimentary bacteria & 2.00 & 2.00 & 2.00 \\
Water column bacteria & 2.00 & 2.00 & 2.00 \\
Water column protozoa & 1.54 & 3.00 & 3.00 \\
\hline
\end{tabular}


when about half of algae net production is consumed by herbivores. Trophic efficiencies of category IIl decrease with decline in coral cover, but the decrease is greatest when grazers increase their total consumption of algal carbon. Trophic efficiencies also highlighted differences among the coral- and algae-dominated states. Efficiencies of trophic categories $\mathrm{V}$ and $\mathrm{VI}$ are lower and higher respectively in the coral-dominated state than in the algae-dominated configurations.

\section{Trophic dependencies}

Dependency coefficients reveal marked differences among the 3 models (Table 4). With loss of coral cover the dependence of all trophic groups on coral-derived carbon (both zooxanthellae and the heterotrophic component), including groups ostensibly remotely connected (e.g. meiofauna, invertivorous fish, carnivorous and detritivorous invertebrates), decreases markedly (Table 4). This result emphasises the fundamental importance of corals in fixing carbon that is utilised by most of the system.

In the transition to increased algal cover, the dependence of second order consumers on algal carbon increases considerably, the only exception being piscivorous fish in the situation where the increase in algal production is lost to the detritus pool. When increases in algal biomass are channeled into the system by increased herbivory (algae-dominated Scenario 2), piscivorous fish become more dependent on algaederived carbon and less dependent on planktivorous fish and zooplankton-derived carbon. Not surprisingly, the importance of grazers (particularly grazing fish) in trophic transfers to most other consumers in the network is greatest when they respond to increased availabilty of algae, e.g. grazing invertebrates become more important and detritivorous and carnivorous invertebrates less important as sources of carbon for invertivorous fish.

Dependency on phytoplankton carbon is relatively low for all groups in all models, although in the algaedominated states transfers to 7.ooplankton from phytoplankton (via direct and indirect circuits) attain nearly $10 \%$ of the total intake. With declining coral cover, dependency of sedimentary microbes, invertebrates and invertivorous fish on phytoplankton carbon declines considerably. Similarly, most groups are less dependent on zooplankton when algae dominate, particularly when grazers respond to the increased availability of algae. In part this reflects the reduced contribution of coral reproductive propagules to zooplankton as coral cover declines

Dependency of higher organisms on flows from microbes bacteria, protozoa, microzooplankton and meiofauna) is low, although clearly there are strong dependencies within the subnetworks of water column microbes and sedimentary microbes. All sedimentary microbes demonstrate strong dependency on carnivorous and detritivorous invertebrates, which make the single largest contribution to the sedimentary detritus pool at the base of the sedimentary microbe subnetwork. The large increase in dependency of water column protozoa and microzooplankton on water column detritus and bacteria for the algae-dominated scenarios reflects that some water column protozoa are imported in the coral-dominated state but imports are not required to balance the algae-dominated networks,

\section{DISCUSSION}

\section{The models: gross properties and comparison with other schemata}

The overriding feature of carbon flux on the shallow front of Davies Reef is the dominance of exogenous inflows and exports, not because of high standing concentrations of carbon in the water column but by virtue of high flow rates and thus transport of large volumes of water Moreover, our estimates of exogenous inputs do not include water moving into the reef front zone and then around the reef without passing over it, which greatly exceeds volumes flowing over the reef flat from the front zone (Hamner \& Hauri 1981, Hamner \& Wolanski 1988, Hamner et al. 1988). Although a portion of the carbon exported from the reef front zone will be utilised by organisms of the reef flat (Ayukai in press), lagoon and back reef, it is clear that the paradigm of low advective inputs and the importance of relatively tight and efficient recycling of inorganic nutrients (e.g. Muscatine \& Porter 1977, Erez 1990, see also D'Elia \& Wiebe 1990) does not apply to all organic elements, and certainly not to organic carbon. Compared to larger temperate marine systems, e.g. the Baltic Sea (Wulff \& Ulanowicz 1989) and Chesapeake Bay, USA (Baird \& Ulanowicz 1989), carbon flux on Davies Reef is dominated to a greater degree by exogenous transfers; but, carbon of exogenous origin comprises a much smaller amount of the total internalised flux, and recycling of carbon is higher on the reef than in these temperate systems ( 26 to $32 \%$ of total flux recycled on Davies Reef versus $22 \%$ in the Chesapeake and Baltic systems).

The models presented here are not directly comparable to previous schemata (cf. Polovina 1984, Wilkinson 1987, Sorokin 1990b) as our models contain a greater number of trophic compartments and there is 
Table 4. Dependency coefficients (\%) for each of the 3 models. These give the fraction (\%) of total carbon intake of one compartment (in the rows) that is contributed by another (in the columns), including direct, indirect and recycled flow's. This is shown as 'Dependency OF $(\downarrow)$ :' groups in rows 'ON: $(\leftarrow \quad \rightarrow)$ ' groups in columns. Top values (normal type) are for the coral-dominated state; middle values (italics) are for the algae-dominated state where the increase in production of algal carbon is lost to detritus (Scenario 1); lower values (bold) are for the algae-dominated state where the increase in production of algal carbon flows to detritus and grazers in equal proportions (Scenario 2). Cpt: Compartment

\begin{tabular}{|c|c|c|c|c|c|c|c|c|c|c|c|c|c|c|c|c|c|c|c|}
\hline $\begin{array}{l}\text { Dependency } \\
\text { OF }(\downarrow) \text { : }\end{array}$ & $\begin{array}{c}\text { Cpt } \\
1\end{array}$ & $\begin{array}{c}\text { Cpt } \\
2\end{array}$ & $\begin{array}{c}\text { Cpt } \\
3\end{array}$ & $\begin{array}{c}\text { Cpt } \\
4\end{array}$ & $\begin{array}{c}\text { Cpt } \\
5\end{array}$ & $\begin{array}{c}\text { Cpt } \\
6\end{array}$ & $\begin{array}{c}\text { Cpt } \\
7\end{array}$ & $\begin{array}{c}\text { Cpt } \\
8\end{array}$ & $\begin{array}{l}\text { ON: } \\
\text { Cpt } \\
9\end{array}$ & $\begin{array}{l}{[\leftarrow} \\
\mathrm{Cpt} \\
10\end{array}$ & $\begin{array}{c}\rightarrow) \\
\mathrm{Cpt} \\
11\end{array}$ & $\begin{array}{l}\text { Cpt } \\
12\end{array}$ & $\begin{array}{c}\text { Cpt } \\
13\end{array}$ & $\begin{array}{c}\text { Cpt } \\
14\end{array}$ & $\begin{array}{l}\text { Cpt } \\
15\end{array}$ & $\begin{array}{l}\mathrm{Cpt} \\
16\end{array}$ & $\begin{array}{l}\text { Cpt } \\
17\end{array}$ & $\begin{array}{c}\text { Cpt } \\
18\end{array}$ & $\begin{array}{l}\text { Cpt } \\
19\end{array}$ \\
\hline $\begin{array}{l}\text { Grazing fish } \\
\text { (Cpt 5) }\end{array}$ & $\begin{array}{l}0.6 \\
0.9 \\
\mathbf{0 . 4}\end{array}$ & $\begin{array}{l}8.6 \\
1.7 \\
\mathbf{0 . 8}\end{array}$ & $\begin{array}{l}48.4 \\
48.9 \\
53.1\end{array}$ & $\begin{array}{l}41.5 \\
41.8 \\
42.5\end{array}$ & $\begin{array}{l}0.1 \\
0.2 \\
0.2\end{array}$ & $\begin{array}{l}0.0 \\
0.0 \\
0.0\end{array}$ & $\begin{array}{r}10.2 \\
2.8 \\
1.3\end{array}$ & $\begin{array}{r}10.3 \\
10.3 \\
4.8\end{array}$ & $\begin{array}{l}0.0 \\
0.0 \\
0.0\end{array}$ & $\begin{array}{l}0.0 \\
0.0 \\
0.0\end{array}$ & $\begin{array}{l}0.0 \\
0.1 \\
0.0\end{array}$ & $\begin{array}{l}0.1 \\
0.1 \\
0.0\end{array}$ & $\begin{array}{l}0.0 \\
0.0 \\
0.0\end{array}$ & $\begin{array}{l}0.0 \\
0.0 \\
0.0\end{array}$ & $\begin{array}{l}0.0 \\
0.0 \\
\mathbf{0 . 0}\end{array}$ & $\begin{array}{l}0.0 \\
0.1 \\
0.0\end{array}$ & $\begin{array}{l}0.1 \\
0.1 \\
0.0\end{array}$ & $\begin{array}{l}3.53 \\
2.4 \\
1.1\end{array}$ & $\begin{array}{l}0.0 \\
0.0 \\
\mathbf{0 . 0}\end{array}$ \\
\hline $\begin{array}{l}\text { Grazing } \\
\text { invertebrates } \\
\text { (Cpt 6) }\end{array}$ & $\begin{array}{l}0.8 \\
0.1 \\
0.1\end{array}$ & $\begin{array}{r}13.3 \\
1.2 \\
0.6\end{array}$ & $\begin{array}{l}80.8 \\
84.8 \\
91.5\end{array}$ & $\begin{array}{l}0.5 \\
3.0 \\
1.4\end{array}$ & $\begin{array}{l}0.4 \\
1.0 \\
1.2\end{array}$ & $\begin{array}{l}0.1 \\
0.2 \\
0.2\end{array}$ & $\begin{array}{r}15.6 \\
1.9 \\
1.0\end{array}$ & $\begin{array}{l}1.7 \\
1.0 \\
0.5\end{array}$ & $\begin{array}{r}11.6 \\
11.6 \\
6.2\end{array}$ & $\begin{array}{l}0.0 \\
0.1 \\
0.0\end{array}$ & $\begin{array}{l}0.2 \\
0.4 \\
0.4\end{array}$ & $\begin{array}{l}0.3 \\
0.6 \\
0.3\end{array}$ & $\begin{array}{l}0.0 \\
0.0 \\
0.0\end{array}$ & $\begin{array}{l}0.0 \\
0.0 \\
\mathbf{0 . 0}\end{array}$ & $\begin{array}{l}0.1 \\
0.1 \\
0.0\end{array}$ & $\begin{array}{l}0.1 \\
0.3 \\
0.1\end{array}$ & $\begin{array}{l}0.1 \\
0.1 \\
\mathbf{0 . 0}\end{array}$ & $\begin{array}{l}20.0 \\
20.0 \\
10.7\end{array}$ & $\begin{array}{l}20.0 \\
20.0 \\
10.8\end{array}$ \\
\hline $\begin{array}{l}\text { Corals } \\
\text { (heterotrophic) } \\
\text { (Cpt 7) }\end{array}$ & $\begin{array}{l}5.1 \\
4.3 \\
4.3\end{array}$ & $\begin{array}{l}84.9 \\
61.9 \\
61.9\end{array}$ & $\begin{array}{l}1.3 \\
7.8 \\
6.7\end{array}$ & $\begin{array}{l}0.8 \\
4.9 \\
4.3\end{array}$ & $\begin{array}{l}0.7 \\
1.7 \\
3.6\end{array}$ & $\begin{array}{l}0.1 \\
0.3 \\
0.6\end{array}$ & $\begin{array}{r}30.5 \\
4.7 \\
4.7\end{array}$ & $\begin{array}{l}8.9 \\
7.8 \\
7.8\end{array}$ & $\begin{array}{l}0.1 \\
0.1 \\
0.1\end{array}$ & $\begin{array}{l}0.1 \\
0.1 \\
\mathbf{0 . 2}\end{array}$ & $\begin{array}{l}0.3 \\
0.7 \\
1.2\end{array}$ & $\begin{array}{l}0.5 \\
1.0 \\
1.0\end{array}$ & $\begin{array}{l}0.0 \\
0.0 \\
0.0\end{array}$ & $\begin{array}{l}0.0 \\
0.0 \\
0.0\end{array}$ & $\begin{array}{l}0.0 \\
0.0 \\
0.0\end{array}$ & $\begin{array}{l}0.3 \\
0.7 \\
0.7\end{array}$ & $\begin{array}{l}0.5 \\
0.4 \\
0.4\end{array}$ & $\begin{array}{l}33.2 \\
32.6 \\
32.6\end{array}$ & $\begin{array}{l}0.1 \\
0.1 \\
0.1\end{array}$ \\
\hline $\begin{array}{l}\text { Zooplankton } \\
\text { (Cpt 8) }\end{array}$ & $\begin{array}{l}6.1 \\
9.0 \\
9.0\end{array}$ & $\begin{array}{l}83.5 \\
16.8 \\
\mathbf{1 6 . 8}\end{array}$ & $\begin{array}{l}1.4 \\
5.7 \\
\mathbf{5 . 1}\end{array}$ & $\begin{array}{l}0.9 \\
3.6 \\
3.3\end{array}$ & $\begin{array}{l}0.8 \\
1.5 \\
3.2\end{array}$ & $\begin{array}{l}0.1 \\
0.2 \\
0.4\end{array}$ & $\begin{array}{l}98.4 \\
27.2 \\
27.2\end{array}$ & $\begin{array}{l}8.8 \\
2.8 \\
2.8\end{array}$ & $\begin{array}{l}0.1 \\
0.2 \\
0.2\end{array}$ & $\begin{array}{l}0.1 \\
0.2 \\
\mathbf{0 . 2}\end{array}$ & $\begin{array}{l}0.3 \\
0.5 \\
\mathbf{1 . 0}\end{array}$ & $\begin{array}{l}0.5 \\
0.8 \\
0.8\end{array}$ & $\begin{array}{l}0.0 \\
0.0 \\
0.0\end{array}$ & $\begin{array}{l}0.0 \\
0.0 \\
\mathbf{0 . 0}\end{array}$ & $\begin{array}{l}0.0 \\
0.0 \\
\mathbf{0 . 0}\end{array}$ & $\begin{array}{l}0.3 \\
0.9 \\
\mathbf{0 . 9}\end{array}$ & $\begin{array}{l}0.5 \\
0.7 \\
\mathbf{0 . 7}\end{array}$ & $\begin{array}{l}34.1 \\
23.1 \\
23.1\end{array}$ & $\begin{array}{l}0.1 \\
0.2 \\
0.2\end{array}$ \\
\hline $\begin{array}{l}\text { Carn/detritiv } \\
\text { invertebrates } \\
\text { (Cpt 9) }\end{array}$ & $\begin{array}{l}4.0 \\
0.7 \\
0.7\end{array}$ & $\begin{array}{r}66.3 \\
5.8 \\
5.8\end{array}$ & $\begin{array}{r}4.2 \\
24.0 \\
20.9\end{array}$ & $\begin{array}{r}2.5 \\
15.0 \\
12.9\end{array}$ & $\begin{array}{r}2.2 \\
5.0 \\
10.8\end{array}$ & $\begin{array}{l}0.7 \\
1.2 \\
2.3\end{array}$ & $\begin{array}{r}78.1 \\
9.4 \\
9.4\end{array}$ & $\begin{array}{l}8.2 \\
4.8 \\
4.8\end{array}$ & $\begin{array}{l}57.7 \\
57.8 \\
\mathbf{5 7 . 8}\end{array}$ & $\begin{array}{l}0.2 \\
0.4 \\
0.5\end{array}$ & $\begin{array}{l}0.9 \\
2.0 \\
3.8\end{array}$ & $\begin{array}{l}1.4 \\
3.2 \\
3.2\end{array}$ & $\begin{array}{l}0.1 \\
0.1 \\
\mathbf{0 . 1}\end{array}$ & $\begin{array}{l}0.0 \\
0.0 \\
0.0\end{array}$ & $\begin{array}{l}0.4 \\
0.4 \\
0.4\end{array}$ & $\begin{array}{l}0.6 \\
1.3 \\
1.3\end{array}$ & $\begin{array}{l}0.4 \\
0.3 \\
0.3\end{array}$ & $\begin{array}{l}99.7 \\
99.7 \\
99.5\end{array}$ & $\begin{array}{l}99.9 \\
99.9 \\
99.8\end{array}$ \\
\hline $\begin{array}{l}\text { Invertivorous } \\
\text { fish } \\
\text { (Cpt 10) }\end{array}$ & $\begin{array}{l}3.1 \\
0.6 \\
0.4\end{array}$ & $\begin{array}{r}50.7 \\
4.5 \\
3.5\end{array}$ & $\begin{array}{l}26.7 \\
41.8 \\
\mathbf{5 2 . 1}\end{array}$ & $\begin{array}{r}1.9 \\
11.5 \\
7.8\end{array}$ & $\begin{array}{l}1.7 \\
3.9 \\
6.6\end{array}$ & $\begin{array}{l}29.9 \\
30.3 \\
45.5\end{array}$ & $\begin{array}{r}59.7 \\
7.2 \\
5.7\end{array}$ & $\begin{array}{l}6.3 \\
3.7 \\
2.9\end{array}$ & $\begin{array}{l}74.0 \\
74.0 \\
58.6\end{array}$ & $\begin{array}{l}0.1 \\
0.3 \\
0.3\end{array}$ & $\begin{array}{l}0.7 \\
1.5 \\
2.3\end{array}$ & $\begin{array}{l}1.1 \\
2.4 \\
1.9\end{array}$ & $\begin{array}{l}0.1 \\
0.1 \\
0.0\end{array}$ & $\begin{array}{l}0.0 \\
0.0 \\
\mathbf{0 . 0}\end{array}$ & $\begin{array}{l}0.3 \\
0.3 \\
0.3\end{array}$ & $\begin{array}{l}0.4 \\
1.0 \\
0.8\end{array}$ & $\begin{array}{l}0.3 \\
0.3 \\
0.2\end{array}$ & $\begin{array}{l}76.3 \\
76.3 \\
60.2\end{array}$ & $\begin{array}{l}76.4 \\
76.4 \\
60.4\end{array}$ \\
\hline $\begin{array}{l}\text { Piscivorous } \\
\text { fish } \\
\text { (Cpt 11) }\end{array}$ & $\begin{array}{l}1.7 \\
2.2 \\
1.1\end{array}$ & $\begin{array}{r}24.1 \\
4.3 \\
2.3\end{array}$ & $\begin{array}{l}38.4 \\
40.8 \\
48.3\end{array}$ & $\begin{array}{l}32.4 \\
33.9 \\
38.0\end{array}$ & $\begin{array}{l}77.6 \\
77.9 \\
88.4\end{array}$ & $\begin{array}{l}0.7 \\
0.8 \\
0.8\end{array}$ & $\begin{array}{r}28.3 \\
7.0 \\
3.7\end{array}$ & $\begin{array}{l}23.9 \\
23.7 \\
12.5\end{array}$ & $\begin{array}{l}1.7 \\
1.7 \\
0.9\end{array}$ & $\begin{array}{l}2.2 \\
2.3 \\
1.5\end{array}$ & $\begin{array}{l}0.1 \\
0.3 \\
0.3\end{array}$ & $\begin{array}{l}20.6 \\
20.6 \\
\mathbf{1 0 . 9}\end{array}$ & $\begin{array}{l}0.0 \\
0.0 \\
0.0\end{array}$ & $\begin{array}{l}0.0 \\
0.0 \\
0.0\end{array}$ & $\begin{array}{l}0.0 \\
0.0 \\
\mathbf{0 . 0}\end{array}$ & $\begin{array}{l}0.1 \\
0.3 \\
0.2\end{array}$ & $\begin{array}{l}0.2 \\
0.2 \\
\mathbf{0 . 1}\end{array}$ & $\begin{array}{r}14.8 \\
12.2 \\
6.4\end{array}$ & $\begin{array}{l}1.7 \\
2.8 \\
0.9\end{array}$ \\
\hline $\begin{array}{l}\text { Planktivorous } \\
\text { fish } \\
\text { (Cpt 12) }\end{array}$ & $\begin{array}{l}5.6 \\
7.0 \\
7.0\end{array}$ & $\begin{array}{l}79.3 \\
14.1 \\
14.1\end{array}$ & $\begin{array}{r}2.0 \\
10.2 \\
8.9\end{array}$ & $\begin{array}{l}1.3 \\
6.5 \\
5.7\end{array}$ & $\begin{array}{l}1.2 \\
2.4 \\
5.1\end{array}$ & $\begin{array}{l}0.2 \\
0.4 \\
0.8\end{array}$ & $\begin{array}{l}93.4 \\
22.8 \\
22.8\end{array}$ & $\begin{array}{l}77.1 \\
76.3 \\
76.3\end{array}$ & $\begin{array}{l}0.1 \\
0.2 \\
\mathbf{0 . 2}\end{array}$ & $\begin{array}{l}0.1 \\
0.2 \\
0.3\end{array}$ & $\begin{array}{l}0.4 \\
0.9 \\
1.7\end{array}$ & $\begin{array}{l}0.7 \\
1.4 \\
1.4\end{array}$ & $\begin{array}{l}0.0 \\
0.0 \\
\mathbf{0 . 0}\end{array}$ & $\begin{array}{l}0.0 \\
0.0 \\
0.0\end{array}$ & $\begin{array}{l}0.0 \\
0.0 \\
\mathbf{0 . 0}\end{array}$ & $\begin{array}{l}0.4 \\
1.0 \\
1.0\end{array}$ & $\begin{array}{l}0.5 \\
0.6 \\
\mathbf{0 . 6}\end{array}$ & $\begin{array}{l}50.5 \\
42.2 \\
42.2\end{array}$ & $\begin{array}{l}0.1 \\
0.2 \\
0.3\end{array}$ \\
\hline $\begin{array}{l}\text { Meiofauna } \\
\text { (Cpt 13) }\end{array}$ & $\begin{array}{l}4.0 \\
0.7 \\
0.7\end{array}$ & $\begin{array}{r}66.4 \\
5.8 \\
5.8\end{array}$ & $\begin{array}{r}4.1 \\
23.9 \\
20.7\end{array}$ & $\begin{array}{r}2.5 \\
15.1 \\
13.0\end{array}$ & $\begin{array}{r}2.2 \\
5.1 \\
10.8\end{array}$ & $\begin{array}{l}0.6 \\
1.1 \\
2.0\end{array}$ & $\begin{array}{r}78.2 \\
9.4 \\
9.4\end{array}$ & $\begin{array}{l}8.2 \\
4.8 \\
4.8\end{array}$ & $\begin{array}{l}57.8 \\
57.8 \\
\mathbf{5 7 . 9}\end{array}$ & $\begin{array}{l}0.2 \\
0.4 \\
0.5\end{array}$ & $\begin{array}{l}0.9 \\
2.0 \\
3.8\end{array}$ & $\begin{array}{l}1.4 \\
3.2 \\
3.2\end{array}$ & $\begin{array}{l}0.1 \\
0.1 \\
0.1\end{array}$ & $\begin{array}{l}13.1 \\
13.1 \\
13.1\end{array}$ & $\begin{array}{l}100 \\
100 \\
100\end{array}$ & $\begin{array}{l}0.6 \\
1.3 \\
1.3\end{array}$ & $\begin{array}{l}0.4 \\
0.3 \\
0.3\end{array}$ & $\begin{array}{l}99.8 \\
99.8 \\
99.7\end{array}$ & $\begin{array}{l}100 \\
100 \\
100\end{array}$ \\
\hline $\begin{array}{l}\text { Sedimentary } \\
\text { protozoa } \\
\text { (Cpt 14) }\end{array}$ & $\begin{array}{l}4.0 \\
0.7 \\
0.7\end{array}$ & $\begin{array}{r}66.4 \\
5.8 \\
\mathbf{5 . 8}\end{array}$ & $\begin{array}{r}4.1 \\
23.9 \\
20.7\end{array}$ & $\begin{array}{r}2.5 \\
15.1 \\
13.0\end{array}$ & $\begin{array}{r}2.2 \\
5.1 \\
10.8\end{array}$ & $\begin{array}{l}0.6 \\
1.1 \\
2.0\end{array}$ & $\begin{array}{r}78.2 \\
9.4 \\
9.4\end{array}$ & $\begin{array}{l}8.2 \\
4.8 \\
4.8\end{array}$ & $\begin{array}{l}57.8 \\
57.8 \\
57.9\end{array}$ & $\begin{array}{l}0.2 \\
0.4 \\
0.5\end{array}$ & $\begin{array}{l}0.9 \\
2.0 \\
\mathbf{3 . 8}\end{array}$ & $\begin{array}{l}1.4 \\
3.2 \\
3.2\end{array}$ & $\begin{array}{l}0.1 \\
0.1 \\
0.1\end{array}$ & $\begin{array}{l}0.0 \\
0.0 \\
0.0\end{array}$ & $\begin{array}{l}100 \\
100 \\
100\end{array}$ & $\begin{array}{l}0.6 \\
1.3 \\
1.3\end{array}$ & $\begin{array}{l}0.4 \\
0.3 \\
0.3\end{array}$ & $\begin{array}{l}99.8 \\
99.8 \\
99.7\end{array}$ & $\begin{array}{l}100 \\
100 \\
100\end{array}$ \\
\hline $\begin{array}{l}\text { Sedimentary } \\
\text { bacteria } \\
\text { (Cpt 15) }\end{array}$ & $\begin{array}{l}4.0 \\
0.7 \\
0.7\end{array}$ & $\begin{array}{r}66.4 \\
5.8 \\
\mathbf{5 . 8}\end{array}$ & $\begin{array}{r}4.1 \\
23.9 \\
20.7\end{array}$ & $\begin{array}{r}2.5 \\
15.1 \\
13.0\end{array}$ & $\begin{array}{r}2.2 \\
5.1 \\
10.8\end{array}$ & $\begin{array}{l}0.6 \\
1.1 \\
2.0\end{array}$ & $\begin{array}{r}78.2 \\
9.4 \\
9.4\end{array}$ & $\begin{array}{l}8.2 \\
4.8 \\
4.8\end{array}$ & $\begin{array}{l}57.8 \\
57.8 \\
57.9\end{array}$ & $\begin{array}{l}0.2 \\
0.4 \\
0.5\end{array}$ & $\begin{array}{l}0.9 \\
2.0 \\
\mathbf{3 . 8}\end{array}$ & $\begin{array}{l}1.4 \\
3.2 \\
3.2\end{array}$ & $\begin{array}{l}0.1 \\
0.1 \\
\mathbf{0 . 1}\end{array}$ & $\begin{array}{l}0.0 \\
0.0 \\
0.0\end{array}$ & $\begin{array}{l}0.4 \\
0.4 \\
0.4\end{array}$ & $\begin{array}{l}0.6 \\
1.3 \\
1.3\end{array}$ & $\begin{array}{l}0.4 \\
0.3 \\
\mathbf{0 . 3}\end{array}$ & $\begin{array}{l}99.8 \\
99.8 \\
99.7\end{array}$ & $\begin{array}{l}100 \\
100 \\
\mathbf{1 0 0}\end{array}$ \\
\hline $\begin{array}{l}\text { Water column } \\
\text { bacteria } \\
\text { (Cpt 16) }\end{array}$ & $\begin{array}{l}4.0 \\
0.7 \\
0.7\end{array}$ & $\begin{array}{r}66.5 \\
5.8 \\
\mathbf{5 . 8}\end{array}$ & $\begin{array}{r}3.9 \\
23.8 \\
20.4\end{array}$ & $\begin{array}{r}2.5 \\
15.1 \\
13.0\end{array}$ & $\begin{array}{r}2.2 \\
5.1 \\
10.9\end{array}$ & $\begin{array}{l}0.4 \\
0.9 \\
1.7\end{array}$ & $\begin{array}{r}78.3 \\
9.4 \\
9.4\end{array}$ & $\begin{array}{l}8.2 \\
4.8 \\
4.8\end{array}$ & $\begin{array}{l}0.2 \\
0.4 \\
0.4\end{array}$ & $\begin{array}{l}0.2 \\
0.4 \\
\mathbf{0 . 5}\end{array}$ & $\begin{array}{l}0.9 \\
2.0 \\
3.8\end{array}$ & $\begin{array}{l}1.4 \\
3.2 \\
3.2\end{array}$ & $\begin{array}{l}0.0 \\
0.0 \\
0.0\end{array}$ & $\begin{array}{l}0.0 \\
0.0 \\
\mathbf{0 . 0}\end{array}$ & $\begin{array}{l}0.0 \\
0.0 \\
0.0\end{array}$ & $\begin{array}{l}0.6 \\
1.3 \\
1.3\end{array}$ & $\begin{array}{l}0.4 \\
0.3 \\
0.3\end{array}$ & $\begin{array}{l}100 \\
100 \\
100\end{array}$ & $\begin{array}{l}0.2 \\
0.4 \\
\mathbf{0 . 4}\end{array}$ \\
\hline $\begin{array}{l}\text { Water column } \\
\text { protozod } \\
\text { (Cpt 17) }\end{array}$ & $\begin{array}{l}1.1 \\
0.7 \\
0.7\end{array}$ & $\begin{array}{r}17.9 \\
5.8 \\
\mathbf{5 . 8}\end{array}$ & $\begin{array}{r}1.1 \\
23.8 \\
20.4\end{array}$ & $\begin{array}{r}0.7 \\
15.1 \\
13.0\end{array}$ & $\begin{array}{r}0.6 \\
5.1 \\
10.9\end{array}$ & $\begin{array}{l}0.1 \\
0.9 \\
1.7\end{array}$ & $\begin{array}{r}21.1 \\
9.4 \\
9.4\end{array}$ & $\begin{array}{l}2.2 \\
4.8 \\
4.8\end{array}$ & $\begin{array}{l}0.0 \\
0.4 \\
\mathbf{0 . 4}\end{array}$ & $\begin{array}{l}0.0 \\
0.4 \\
0.5\end{array}$ & $\begin{array}{l}0.2 \\
2.0 \\
3.8\end{array}$ & $\begin{array}{l}0.4 \\
3.2 \\
3.2\end{array}$ & $\begin{array}{l}0.0 \\
0.0 \\
\mathbf{0 . 0}\end{array}$ & $\begin{array}{l}0.0 \\
0.0 \\
0.0\end{array}$ & $\begin{array}{l}0.0 \\
0.0 \\
\mathbf{0 . 0}\end{array}$ & $\begin{array}{r}26.9 \\
100 \\
100\end{array}$ & $\begin{array}{l}0.1 \\
0.3 \\
0.3\end{array}$ & $\begin{array}{l}26.9 \\
100 \\
100\end{array}$ & $\begin{array}{l}0.1 \\
0.4 \\
\mathbf{0 . 4}\end{array}$ \\
\hline $\begin{array}{l}\text { Water column } \\
\text { detritus } \\
\text { (Cpt 18) }\end{array}$ & $\begin{array}{l}4.0 \\
0.7 \\
0.7\end{array}$ & $\begin{array}{r}66.5 \\
5.8 \\
5.8\end{array}$ & $\begin{array}{r}3.9 \\
23.8 \\
20.4\end{array}$ & $\begin{array}{r}2.5 \\
15.1 \\
13.0\end{array}$ & $\begin{array}{r}2.2 \\
5.1 \\
10.9\end{array}$ & $\begin{array}{l}0.4 \\
0.9 \\
1.7\end{array}$ & $\begin{array}{r}78.3 \\
9.4 \\
9.4\end{array}$ & $\begin{array}{l}8.2 \\
4.8 \\
4.8\end{array}$ & $\begin{array}{l}0.2 \\
0.4 \\
0.4\end{array}$ & $\begin{array}{l}0.2 \\
0.4 \\
0.5\end{array}$ & $\begin{array}{l}0.9 \\
2.0 \\
3.8\end{array}$ & $\begin{array}{l}1.4 \\
3.2 \\
3.2\end{array}$ & $\begin{array}{l}0.0 \\
0.0 \\
0.0\end{array}$ & $\begin{array}{l}0.0 \\
0.0 \\
\mathbf{0 . 0}\end{array}$ & $\begin{array}{l}0.0 \\
0.0 \\
\mathbf{0 . 0}\end{array}$ & $\begin{array}{l}0.6 \\
1.3 \\
\mathbf{1 . 3}\end{array}$ & $\begin{array}{l}0.4 \\
0.3 \\
\mathbf{0 . 3}\end{array}$ & $\begin{array}{r}26.6 \\
6.0 \\
6.0\end{array}$ & $\begin{array}{l}0.2 \\
0.4 \\
0.4\end{array}$ \\
\hline $\begin{array}{l}\text { Sedimentary } \\
\text { detritus } \\
\text { (Cpt 19) }\end{array}$ & $\begin{array}{l}4.0 \\
0.7 \\
0.7\end{array}$ & $\begin{array}{r}66.4 \\
5.8 \\
5.8\end{array}$ & $\begin{array}{r}4.1 \\
23.9 \\
20.7\end{array}$ & $\begin{array}{r}2.5 \\
15.1 \\
13.0\end{array}$ & $\begin{array}{r}2.2 \\
5.1 \\
\mathbf{1 0 . 8}\end{array}$ & $\begin{array}{l}0.6 \\
1.1 \\
2.0\end{array}$ & $\begin{array}{r}78.2 \\
9.4 \\
9.4\end{array}$ & $\begin{array}{l}8.2 \\
4.8 \\
4.8\end{array}$ & $\begin{array}{l}57.8 \\
57.8 \\
57.9\end{array}$ & $\begin{array}{l}0.2 \\
0.4 \\
0.5\end{array}$ & $\begin{array}{l}0.9 \\
2.0 \\
3.8\end{array}$ & $\begin{array}{l}1.4 \\
3.2 \\
3.2\end{array}$ & $\begin{array}{l}0.1 \\
0.1 \\
0.1\end{array}$ & $\begin{array}{l}0.0 \\
0.0 \\
0.0\end{array}$ & $\begin{array}{l}0.4 \\
0.4 \\
\mathbf{0 . 4}\end{array}$ & $\begin{array}{l}0.6 \\
1.3 \\
\mathbf{1 . 3}\end{array}$ & $\begin{array}{l}0.4 \\
0.3 \\
\mathbf{0 . 3}\end{array}$ & $\begin{array}{l}99.8 \\
99.8 \\
\mathbf{9 9 . 7}\end{array}$ & $\begin{array}{l}57.8 \\
57.9 \\
\mathbf{5 7 . 9}\end{array}$ \\
\hline
\end{tabular}


no, or only partial, overlap in the identity of most compartments. However, some useful general comparisons can be made. In terms of gross trophic structure, the ECOPATH model developed for French Frigate Shoals in Hawaii, USA (Polovina 1984, Atkinson \& Grigg 1984, Grigg et al. 1984), demonstrates some similarity to our models in that there is sorne resolution of higher order consumers (however, the top-down approach of ECOPATH is quite different to that employed here). Polovina's (1984) species group of 'reef fishes' closely parallels the sum of the fish groups in our models However, estimates of the total production of reef fish in our models are over an order of magnitude greater than that predicted by ECOPATH, despite that in both models these fish are feeding at about the same trophic level and that the ecological efficiency of trophic category II is lower on Davies Reef $(\sim 6 \%)$ than at French Frigate Shoals (20\%). This is explained in part by an order of magnitude higher standing biomass of fish (Williams \& Hatcher 1983) and higher benthic primary production (algae + corals; see Appendix 1) on midshelf reefs in the GBR. Also, in ECOPATH only $-5 \%$ of net primary production (NPP) reaches non-benthic predators (Atkinson \& Grigg 1984), whereas in our models at least 16.1 to $26.9 \%$ of NPP (depending on the model) is utilised by predators (invertivorous fish. piscivorous fish and zooplankton) feeding at trophic level 3. That so little plant-derived carbon reaches higher consumers in the ECOPATH scenario reflects the low trophic efficiencies of trophic categories III and IV $(4$ and $2 \%$ in ECOPATH versus -17 and $18 \%$ respectively for the coral-dominated state on Davies Reef). These differences suggest that many more data are required on flux rates, assimilation efficiencies and other physiological processes at a variety of appropriate temporal and spatial scales and, also, that a critical comparison of top-down and bottom-up approaches to modelling material fluxes is warranted.

Although the overall structure and trophic resolution of Sorokin's (1990b) general model of energy flow on a 'typical' coral reef is simpler than in our models, fundamental differences in the role of bacteria in the 2 schemes warrant discussion. In Sorokin's model detritus is the foundation of the system, and the bulk of primary production enters the network via microbial links. Bacteria account for $>70 \%$ of the total energy flow, and the second greatest flow is zooxanthellae $\rightarrow$ coral $\rightarrow$ detritus. In our model, the total flux of carbon from sedimentary and water column detritus to heterotrophs $\left(13.5 \mathrm{~g} \mathrm{C} \mathrm{m}^{-2} \mathrm{~d}^{-1}\right)$ is similar to the transfer from zooxanthellae to corals $\left(12.6 \mathrm{~g} \mathrm{C} \mathrm{m}^{-2} \mathrm{~d}^{-1}\right)$, but most of the flux from detritus to heterotrophs is accounted for by corals feeding on POC $(49 \%)$ and by detritivores $(48 \%)$, not by bacteria $(1.7 \%)$. Note that whereas a large proportion $(50 \%)$ of water column bacteria on coral reefs may be attached to detrital particles (Moriarty 1979), they likely amount to only a negligible portion $(<0.1 \%)$ of POC (Coffroth 1990). Also, dependency coefficients (Table 4 ) suggest that flows via bacteria may be relatively unimportant for higher order consumers

This discrepancy cannot be accounted for by the different units of the models; how then can such a large and fundamental difference be reconciled? Part of the answer is that Sorokin's estimates of bacterial biomass and production are too high (Ducklow 1990) and that his model is for an entire reef system and not a specific zone dominated by hard substratum. Unlike other reef zones, particularly lagoonal areas, there is relatively little sediment on the shallow front zone of Davies Reef (at $7.5 \mathrm{~m}$ only $5 \%$ of the benthos is sand; Daniel et al. 1985, Klumpp et al. 1987). Our estimates of total bacterial metabolism are likely to be underestimates because we were unable to take into account utilisation of DOC or the activity of bacteria on hard substrata or on biological surfaces. However, even if we assume that bacterial production is as high on hard substrata (excluding live coral) as it is on sand, our conclusions are unchanged; given $41 \%$ cover of hard substratum with surface rugosity of 1.86 (Klumpp \& McKinnon 1989), if bacterial production on this substratum is equivalent to that on sand on an areal basis, then the proportion of total flux from detritus $\rightarrow$ heterotrophs via bacteria increases from 1.7 to $2.0 \%$, which is negligible. Moreover, since much of the hard substrata is coralline algae, which characteristically has regions of thallus with low densities of bacteria (Johnson et al. 1991a, b), the assumption of equivalent production of bacteria on hard substrata as on sand may be an overestimate. Spatial variation in the biomass and production of water column bacteria is unlikely to account for the discrepancy since standing stocks $\left(11.9 \mathrm{mg} \mathrm{C} \mathrm{m}{ }^{-3}\right.$ for Davies Reef; $\mathrm{T}$. Ayukai unpubl.) are low and do not vary greatly among zones (Moriarty et al. 1985a, Ducklow 1990). Thus, our cautious conclusion is that the importance of microbial links in recycling and flows to heterotrophy are highly spatially variable on coral reefs and appear to be relatively unimportant in areas dominated by corals and strong hydrodynamic flow but much more important in areas with high cover of sediments. However, we add the caveat and echo Sorokin's (1990b) sentiments that much more work is required on the importance of microbes as a food source.

\section{Effects of transition to an algae-dominated state}

Extensive replacement of live coral with free-living algae can lead to net erosion. of reefs as a result 
of depletion of reef-building organisms (Bak 1990, Glynn \& Colgan 1992), but the effects of shifts in community structure of this kind on carbon flux and other aspects of system function are poorly understood. Unless loss of corals is extreme, the same kinds of trophic groups and qualitatively identical linkages between them describe both community states, although the precise species complement of any particular trophic group may differ The question arises whether a particular trophic group functions similarly regardless of its precise species signature (the 'redundancy' hypothesis) or whether changes in the species complement of particular trophic groups incrementally affect the system (the 'rivet' hypothesis; Chapin et al. 1992). Our results show that, even at the coarse level of trophic structure considered in our networks, network properties and the structure of transfers of carbon among trophic compartments in terms of both absolute and relative fluxes are fundamentally affected by shifts in community structure. Moreover, our analysis is conservative in that it assumes no changes in the diversity or relative composition of any trophic compartment other than corals.

Replacement of corals by algae realises decreases in primary productivity, and thus decreases in overall system size and activity (e.g. in TST and $A$ ), although there is an increase in the magnitude of transfers to detritus. The significant decrease in system size is not dependent on our assumption that algae-covered substrata on coral- and algae-dominated reefs are similarly productive, since even with the most optimistic estimates of algal production in the algaedominated state (see 'Methods'), loss of coral cover realises a large decrease in system size. Decline in size and ascendancy is normally indicative of disturbance to, or stress on, a system (Kay et al. 1989). However, the structure of recycling suggests that the relative magnitude of the 'stress' is not large. Disturbed systems tend toward fewer cycles, short circuit lengths and a preponderance of single-nexus cycles (Baird \& Ulanowicz 1989); but, our networks reveal moderate numbers of cycles, including some with long circuit lengths and some large nexuses. Increase in the proportion of total flux that is recycled, as was observed with loss of coral cover, has been reported for other disturbed marine systems (Ulanowicz 1984, Baird \& Ulanowicz 1989).

The decrease in TST and A occurs largely because, in our models, productivity of live coral is greater than that of equivalent dead coral overgrown with epilithic algae. Since this difference in productivity underscores many of the differences in network characteristics between the 2 states, the flux estimates for gross primary production of zooxanthellae and epilithic algae warrant careful scrutiny. Our values for algal production come from extensive and repeatable measurements at Davies Reef (Klumpp \& McKinnon. 1989, 1992). Estimates for Davies Reef are nearly identical to those for a variety of other reefs in the GBR system (Klumpp \& McKinnon 1992), but may be lower than those for equivalent algal assemblages in the Caribbean (Carpenter 1985). Our value for coral production is more problematic (gross production = $27 \mathrm{~g} \mathrm{C} \mathrm{m}^{-2}$ coral $\mathrm{d}^{-1}=14.8 \mathrm{~g} \mathrm{C} \mathrm{m}^{-2}$ substratum $\mathrm{d}^{-1}$; Appendix 1), and is midrange of several estimates for coral outcrops on the GBR (Kinsey 1985). This level of production exceeds the high end of the range suggested by Muscatine $\left(1990 ; 14 \mathrm{~g} \mathrm{C} \mathrm{m}^{-2} \mathrm{~d}^{-1}\right)$, but is similar to the high rates recorded for Pocillopora damicornis (28.9 $\mathrm{g} \mathrm{C} \mathrm{m}^{-2} \mathrm{~d}^{-1}$ ) by Jokiel \& Morrissey (1986). Smith (1981) asserts that rates of gross production of the order of $25 \mathrm{~g} \mathrm{C} \mathrm{m}^{-2} \mathrm{~d}^{-1}$ can be found in shallow thickets of coral. Our point is that, while Kinsey's (1985) values for productivity of coral outcrops (which will include some algal production) are at the high end of production rates expected of corals, even if these estimates are high by $100 \%$, the same qualitative behaviour in network characteristics would result and our conclusions would not change. For there to be no change in total gross primary production in the shift to dominance by algae, production by coral zooxanthellae would need to be greater than an order of magnitude lower than the estimate used here, which is clearly untenable. Our estimate of total gross primary production (corals + algae $=15.9 \mathrm{~g} \mathrm{C} \mathrm{m}^{-2} \mathrm{~d}^{-1}$ for the coral-dominated state) is within the normal range for coral-dominated reef areas (e.g. see Smith 1981 , Adey 1983, Kinsey 1985).

The shift in primary production from domination by zooxanthellae to domination by free-living algae markedly alters the relative importance of pathways in the network, e.g. herbivorous fish and invertebrates account for $2.2 \%$ of net primary production (NPP) and coral heterotrophy for $90.3 \%$ of NPP when corals dominate, but when algae dominate and grazers respond to increased availability of algae, herbivores and corals account for 36.3 and $24.5 \%$ of NPP respectively. Changes in flow structure with loss of corals are indicated by an increased average path length, reduced average trophic level of most of the second order consumers, reduced trophic (ecological) efficiencies of most trophic categories, and marked changes in dependencies on other trophic groups. An unequivocal conclusion is that in the coral-dominated state, carbon fixed by zooxanthellae is used indirectly by most organisms in the system, even those seemingly remotely connected. This is revealed by both the dependency coefficients, which emerge as the measures most sensitive to changes in commu- 
nity structure, and the changes in the effective trophic level of higher order consumers.

\section{Carbon flux in the algae-dominated state}

The precise details of changes in network properties with loss of coral cover depend on the fate of the additional algae-derived carbon in the system, but published empirical data on this aspect are currently lacking. We considered situations in which the increased production of algae is lost to detritus and grazers do not respond (Scenario 1), and alternatively, where consumption rates of algae increase (Scenario 2). It is possible that either scenario may apply, although the balance of evidence suggests that on the GBR grazing fish do not respond to the increase in algal biomass with loss of coral cover. Williams (1986) examined the effect of increased cover of algae (caused by outbreaks of crown-ofthorns starfish) on grazing fish on several GBR reefs by comparing population abundance estimates made 3 yr prior to starfish infestation with estimates made soon after destruction of corals. Although Williams found that temporal differences in fish populations on impacted reefs with high cover of algae were not detectably different to those on control reefs dominated by corals, his results are equivocal because his tests have low power (given a $\log _{5}$ scale measure of abundance and low replication), the time between loss of coral cover and censusing of fish is likely to have been too short for changes in fish population size to occur, and his methods could not detect individual functional responses such as changes in grazing, growth or fecundity rates. However, more detailed recent work has corroborated Williams' conclusions. A study of 6 GBR reefs found that the abundance, biomass, feeding rates, growth rates, and gonad indices of grazing fish are similar on algaedominated reefs impacted by crown-of-thorns starfish and coral-dominated reefs ( $T$ Hart unpubl. data). These findings suggest that grazing fish on the GBR may be recruitment limited (see Doherty \& Williams 1989) and not food limited However, this situation is unlikely to apply to all reef systems. In direct contrast to results for the central GBR, Robertson (1991) recorded increases of 250 and $160 \%$ in the population sizes of 2 species of acanthurid in response to increased availability of algae after die off of sea urchins in Panama. Since there was no change in recruitment rates of the fish during the study, Robertson (1991) concluded that the increase in population sizes indicated food limitation.

The response of grazers, both fish and invertebrates, to changes in absolute and relative abundances of algae and corals requires further resolution. An important component of this question is the response of herbivores to different kinds of algae since in some systems the community structure of algal assemblages changes during the phase transition (Carpenter 1990. Hughes 1994) and different herbivores respond differentially to different algal species (e.g. Hackney et al. 1989, Padilla 1989, Hay 1991). This information is important to the question of network properties since, although the network is considerably more sensitive to wholesale shifts in structure than to our alternative scenarios of the fate of photosynthetically fixed carbon in the algae-dominated state, the 2 scenarios for the algae-dominated state yielded dissimilar values for some parameters. Notably, trophic dependence of higher order consumers on grazers, flow diversity (a log scale), the relative importance of recycling, trophic efficiency of trophic category III, and the effective trophic level of piscivorous fish were sensitive to the relatively small differences in the fate of algal carbon.

This study has shown how community parameters can affect an aspect of ecosystem functioning on a coral reef. Given the diversity of reef forms and variability among reefs in flux processes (e.g. Hatcher 1990), our results are unlikely to apply to all coral reefs. Furthermore, despite the appeals of workers over a decade ago to give more emphasis to whole reef systems and not just to convenient components of them (e.g. Hatcher 1983b), there remains a dearth of system-level information about coral reefs and a poor understanding of how population and community parameters relate to functions at the system level. Given the intensity and extent of anthropogenic impact on coral reef systems (Wilkinson 1993), there is urgent need for this situation to be rectified. An integrated, multidisciplinary and largescale manipulative (intentional or otherwise) study at the system level will go a considerable way towards achieving this goal.

Acknowledgements. We are grateful to Dan Alongi, Tenshi Ayukar, Kerry Black and Tony Hart for providing their unpublished data, to D. Alongi and $T$ Ayukar for discussion of critical aspects of the models, and to Chris Crossland for comments on an early draft. Several of the ideas presented benefitted from discussion with participants in the SCOPE workshop on coral reefs (Florida, November 1993). We thank Tenshi Ayukar, Ken Mann, Fred Wulf and particularly Bruce Hatcher for constructive reviews. We also thank Robert Peters for information on physiological parameters for marine invertebrates and the captain and crew of the RV 'Lady Basten' for providing a suitable environment for the beginnings of the work. The project was jomtly funded by AIMS. grants to C. Johnson from the University of Queensland, and a Core Program FRD grant to J. Field, G. Branch and C. Griffith. This is AIMS contribution number 616 . 
Appendix 1. Sources and/or derivation of estimates of carbon flux ( $\mathrm{g} \mathrm{m}^{-2} \mathrm{~d}^{-1}$ ) on the front slope of Davies Reef (depth 5 to $10 \mathrm{~m}$, mean depth $7.5 \mathrm{~m}$ ) when unaffected by crown-ot-thorns starfish Acanthaster planci and coral cover is $55 \%$. Flows are calculated assuming steady state for each compartment and for the system as a whole Flows $X \rightarrow Y$ indicate flows from compartment $X$ to compartment $Y$ The number of decimal places does not indicate the precision of the estimates but art: required in some places to balance flows. Exogenous imports consider only water that eventually flows over the reef and not that which flows parallel to the front and around the reef to exit downstream (see 'Methods' for details). AE: assimulation efficiency; $C$ : consumption, COA coralline algae; $d w$ : dry weight; EAC: epilithic algal community: fw: fresh weight; $P_{G}$ : gross production, $P_{N}$ : net production; $R$ : Iespiration; $B$ : biomass

\begin{tabular}{|c|c|c|c|}
\hline Compartment & Flow & $\begin{array}{l}\text { Magnitude of flow } \\
\qquad\left(g \mathrm{Cm}^{-2} \mathrm{~d}^{-1}\right)\end{array}$ & Source/denvation \\
\hline \multirow[t]{5}{*}{$\begin{array}{l}\text { Phytoplankton } \\
\text { (Cpt 1) }\end{array}$} & Imported & 97.20 & 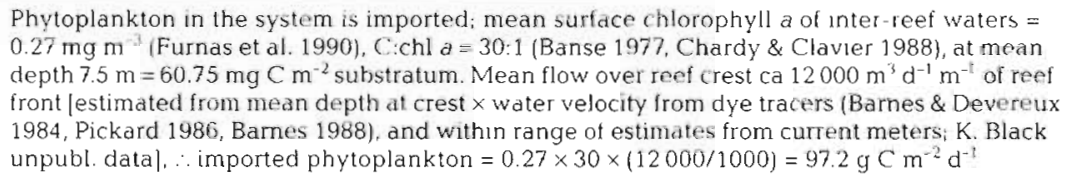 \\
\hline & $1 \rightarrow 7$ & 0.74 & $\begin{array}{l}\text { Total consumption of POC by corals }=7.4 \mathrm{~g} \mathrm{C} \mathrm{m}^{-2} \mathrm{~d}^{-1} \text { and of this } 6.6 \mathrm{~g} \mathrm{C} \mathrm{m}^{-2} \mathrm{~d}^{-1} \text { is from } \\
\text { detritus (see Cpt 18) and } 0.06 \text { from protozoa and other microzooplankton in the water } \\
\text { column (see Cpt 19), . by difference flow to corals }=0.74 \mathrm{~g} \mathrm{C} \mathrm{m}^{-2} \mathrm{~d}^{-1} \text { This cstımate for } \\
\text { phytoplanktor }>2 \mu \mathrm{m}=10 \% \text { of POC available for corals (assummg corals ar: unable to feed } \\
\text { on particles }<2 \mu \mathrm{m} \text { ), which is close to that of } 8.3 \% \text { of POC estimated tor Davies Reef lagoon } \\
\text { (Roman et al. } 1990 \text { ) }\end{array}$ \\
\hline & $1 \rightarrow 8$ & 0.029 & $\begin{array}{l}\text { Biomass-specific consumption of phytoplankton by zooplankton }=1.728 \mathrm{mg} \mathrm{C}^{\left(\mathrm{mg}^{-1} \mathrm{C} \mathrm{d}\right.}{ }^{1} \\
\text { (from Roman et al. 1990), } \therefore \text { total consumption }=1.728 \times 16.6=0.029 \mathrm{~g} \mathrm{C} \mathrm{m}^{-2} \mathrm{u}^{\mathrm{u}} \text { (biomass of } \\
\text { zooplankton from Cpt 8) }\end{array}$ \\
\hline & $R$ & 0 & Respuration $=0$ since all phytoplankton is regarded as ımported \\
\hline & Exported & 96.431 & By difference \\
\hline \multirow[t]{3}{*}{$\begin{array}{l}\text { Corals: } \\
\text { zooxanthellae } \\
\text { (Cpt 2) }\end{array}$} & $P_{\mathrm{Ci}}$ & 14.8 & $\begin{array}{l}P_{c} \text {, from photosynthesis of corals }=27 \mathrm{~g} \mathrm{C} \mathrm{m}^{-2} \mathrm{~d}^{-1} \text { (midrange of values for coral outcrops at } \\
6 \text { sites on the GBR; Kunsey 1985). Adjusting to take into account that coral cover on the reef } \\
\text { stope at ca } 7.5 \mathrm{~m} \text { is } 55 \% \text { (Daniel et al. 1985), overall } P_{\mathrm{G}}=14.8 \mathrm{~g} \mathrm{C} \mathrm{m}^{-2} \mathrm{~d}^{-1} \text {. See 'Discussion' } \\
\text { for evaluation of these estimates }\end{array}$ \\
\hline & $2 \rightarrow 7$ & 12.58 & $\begin{array}{l}\text { Carbon translocated from zooxanthellae to the animal host }=85 \% \text { of carbon fixed Imidrunge } \\
\text { of values from Davies ( } 1984) \text {. McCloskey \& Muscatine }(1984) \text {, and Edmunds \& Davies } \\
\text { (1986) for shallow corals } 3 \text { to } 10 \mathrm{~m} \text { depth }=12.58 \mathrm{~g} \mathrm{C} \mathrm{m}^{-2} \mathrm{~d}^{-1}\end{array}$ \\
\hline & $R$ & 2.22 & 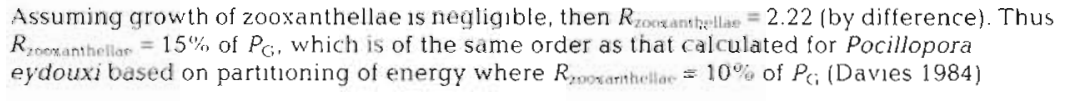 \\
\hline \multirow[t]{4}{*}{$\begin{array}{l}\text { Turf algae } \\
\text { (Cpt 3) }\end{array}$} & $P_{\mathrm{i}}$ & 0.65 & $\begin{array}{l}P_{\mathrm{G}} \text { of the EAC averaged across seasons }=1.43 \mathrm{~g} \mathrm{C} \mathrm{m}^{-2} \mathrm{~d}^{-1} \text { (Klumpp \& McKinnon 1989); } \\
\text { since EAC accupies } 41 \% \text { of reef slope area (Klumpp \& McKinnon 1989) and adjusting for } \\
\text { reef surface rugosity factor of } 1.86 \text { for reef slope (Klumpp \& McKinnon 1989), } P_{\mathrm{C}} \text { EAC } \\
\text { overall }=1.08 \mathrm{~g} \mathrm{C} \mathrm{m}^{-2} \mathrm{~d}^{-1} \text {. Since } 60 \% \text { of EAC is turf (Klumpp \& McKinnon 1989), } P_{\mathrm{G}} \text { turf = } \\
0.6 \times 1.08=0.65 \mathrm{~g} \mathrm{C} \mathrm{m}^{-2} \mathrm{~d}^{-1} \text { and } P_{\mathrm{C}} \text { COA }=0.4 \times 1.08=0.43 \mathrm{~g} \mathrm{C} \mathrm{m}^{-2} \mathrm{~d}^{-1}\end{array}$ \\
\hline & $\begin{array}{l}3 \rightarrow 5 \\
3 \rightarrow 6\end{array}$ & $\begin{array}{l}0.14 \\
0.04\end{array}$ & $\begin{array}{l}\text { From } P_{\mathrm{G}} \text { and } R, P_{\mathrm{N}} \text { for EAC is calculated as } 0.6 \mathrm{~g} \mathrm{C} \mathrm{m}^{-2} \mathrm{~d}^{-1} \text { (rounded from } 0.59 \text {; see below } \\
\text { for calculations of } R \text {, and ca } 50 \% \text { of } P_{\mathrm{N}} \text { is lost to grazers (Hatcher } 1983 \mathrm{a} \text {. Klumpp \& Polunin } \\
1990, \text { Klumpp \& McKinnon } 1992 \text { ), } \therefore 0.3 \mathrm{~g} \mathrm{C} \mathrm{m}^{-2} \mathrm{~d}^{-1} \text { is grazed. Now, } 60 \% \text { of EAC is turf algae } \\
\text { thus } 0.6 \times 0.3=0.18 \mathrm{~g} \mathrm{C} \mathrm{m}^{-2} \mathrm{~d}^{-1} \text { of turf is lost to all grazers, including both fish and inverte- } \\
\text { brates, and of this ca } 80 \%\left(0.8 \times 0.18=0.14 \mathrm{~g} \mathrm{C} \mathrm{m}^{-2} \mathrm{~d}^{-1} \text { ) is accounted for by fish and the }\right. \\
\text { remainder }\left(0.04 \mathrm{~g} \mathrm{C} \mathrm{m}^{-2} \mathrm{~d}^{-1} \text { ) by macroinvertebrates (derived from Hatcher } 1983 \mathrm{a} \text {, Klumpp \& }\right. \\
\text { Pulfrich } 1989, \mathrm{Klumpp} \& \text { Polunin } 1990 \text { ). Assume all of losses of COA }(=40 \% \text { EAC) are to } \\
\text { fish, which amounts to } 0.4 \times 0.3=0.12 \mathrm{gC} \mathrm{m} \mathrm{g}^{-2} \mathrm{~d}^{-1}\end{array}$ \\
\hline & $3 \rightarrow 18$ & 0.18 & By difference \\
\hline & $R$ & 0.29 & $\begin{array}{l}P_{\mathrm{G}}: R \text { for total EAC }=2.2: 1 \text { (Klumpp \& McKinnon 1989), . R }=0.49 \mathrm{~g} \mathrm{C} \mathrm{m}^{-2} \mathrm{~d}^{-1} \text { for total EAC } \\
\text { and } 0.6 \times 0.49=0.29 \mathrm{~g} \mathrm{C} \mathrm{m}^{-2} \mathrm{~d}^{-1} \text { for the turf component and } 0.4 \times 0.49=0.2 \mathrm{~g} \mathrm{C} \mathrm{m}^{-2} \mathrm{~d}^{-1} \text { for COA }\end{array}$ \\
\hline $\mathrm{COA}$ & $P_{\mathrm{G}}$ & 0.43 & See calculations for turf algae (Cpt 3 ) \\
\hline \multirow{3}{*}{ (Cpt 4) } & $4 \rightarrow 5$ & 0.12 & See calculations for turf algae (Cpt 3) \\
\hline & $4 \rightarrow 18$ & 0.11 & By difference \\
\hline & $R$ & 0.20 & See calculations for turf algae (Cpt 3$)$ \\
\hline $\begin{array}{l}\text { Grazing fish } \\
\text { (Cpt 5) }\end{array}$ & $\begin{array}{l}5 \rightarrow 8 \\
5 \rightarrow 11\end{array}$ & $\begin{array}{l}0.001 \\
0.161\end{array}$ & 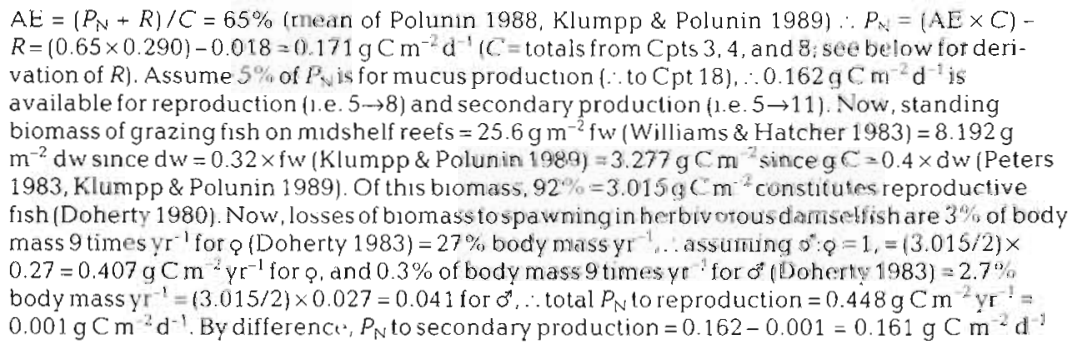 \\
\hline
\end{tabular}


Appendix 1, continued

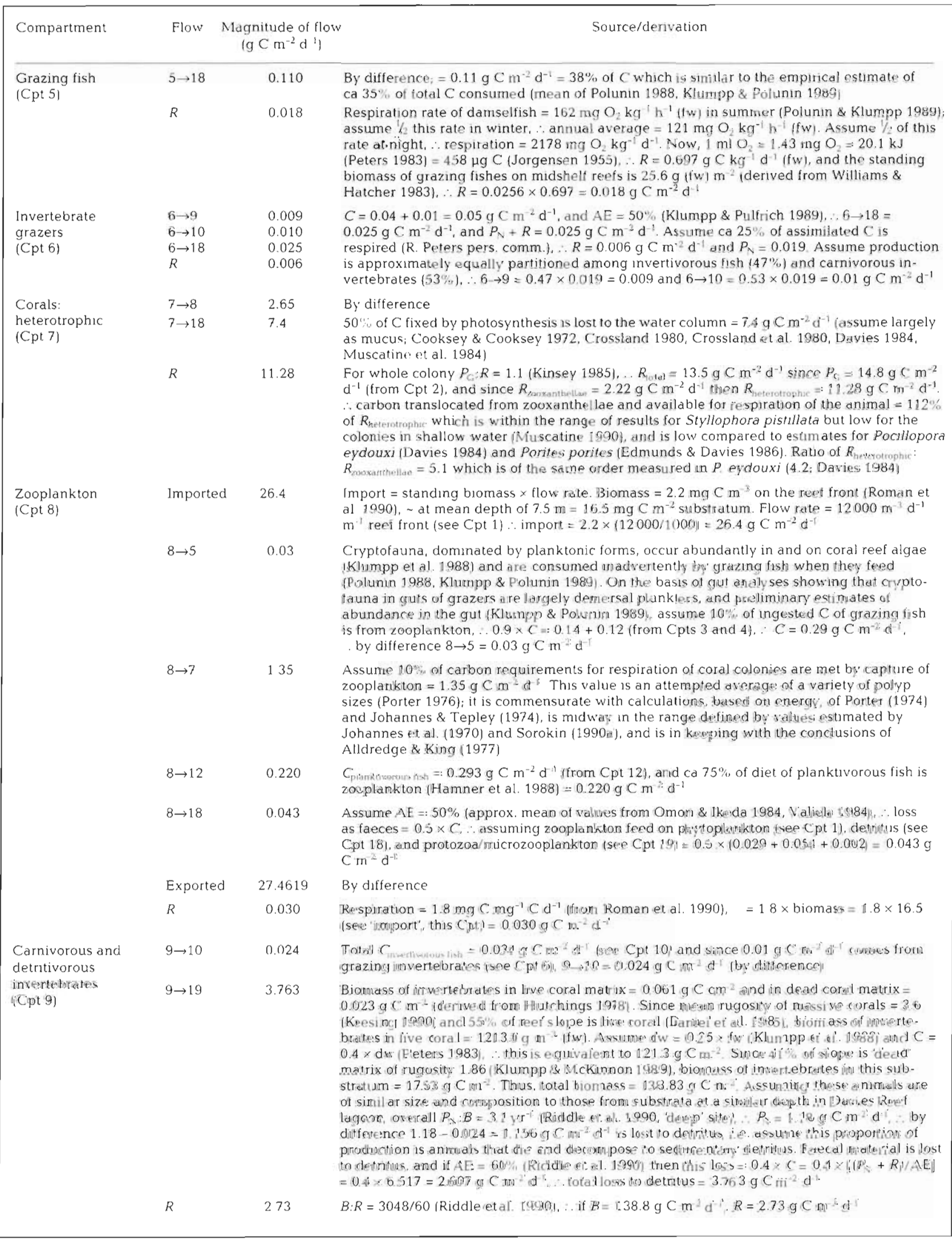


Appendix 1, continued

\begin{tabular}{|c|c|c|c|}
\hline Compartment & Flow Ma & $\begin{array}{l}\text { itude of flaw } \\
\left.C m^{-2} d^{-1}\right)\end{array}$ & Source/derivation \\
\hline \multirow[t]{4}{*}{$\begin{array}{l}\text { Invertivorous fish } \\
\text { (Cpt 10) }\end{array}$} & $10 \rightarrow 8$ & 0.0004 & 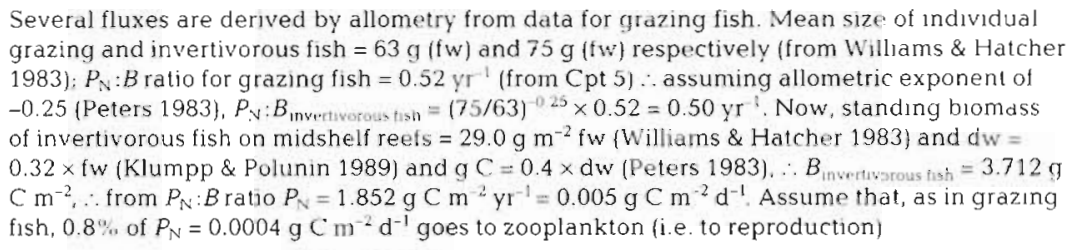 \\
\hline & $10 \rightarrow 11$ & 0.0046 & The remainder of production $=0.0046 \mathrm{~g} \mathrm{C} \mathrm{m}^{-2} \mathrm{~d}^{-1}$ is lost to piscivorous fish \\
\hline & $10 \rightarrow 18$ & 0.010 & $\begin{array}{l}C=\left(P_{\mathrm{N}}+R\right) / \mathrm{AE} \ldots \text { assuming } \mathrm{AE}=70 \% \text {, which is slightly greater than for tish feeding on } \\
\text { algae, } C=10.005+0.019) / 0.7=0.034 \mathrm{~g} \mathrm{C} \mathrm{m}^{-2} \mathrm{~d}^{-1} \text {, and assume that since } \mathrm{AE}=70 \% \text { then } \\
30 \% \text { of } C=0.010 \mathrm{~g} \mathrm{Cm}^{-2} \mathrm{~d}^{-1} \text { is lost as faeces to column detritus }\end{array}$ \\
\hline & $R$ & 0.019 & 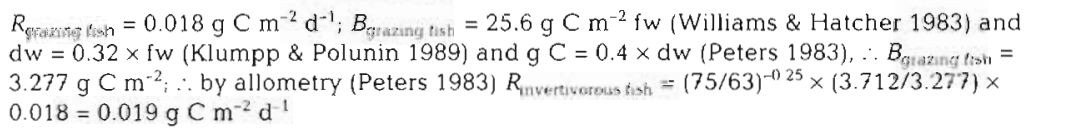 \\
\hline \multirow[t]{4}{*}{$\begin{array}{l}\text { Piscivorous fish } \\
\text { (Cpt 11) }\end{array}$} & $11 \rightarrow 8$ & 0.0002 & $\begin{array}{l}\text { Note: as for Cpts } 10 \text { and } 12 \text {, reliance is made of emprical estimates for grazing fish) } \\
\left.C=0.161+0.0046+0.0427=0.2083 \mathrm{~g} \mathrm{C} \mathrm{m}^{-2} \mathrm{~d}^{-1} \text { (from Cpts } 5,10 \text { and } 12\right) ; C=\left(P_{\mathrm{N}}+R\right) / \mathrm{AE} \\
\text { assuming } \mathrm{AE}=70 \%\left(8 \% \text { higher than that of grazing fish) then } P_{\mathrm{N}}+R=0.146 ; \text { assume }\right. \\
\text { that, as in grazing fish, } P_{\mathrm{N}}: R=0.26 \text {, then } P_{\mathrm{N}}=0.030 \text { and } R=0.116 \mathrm{~g} \mathrm{C} \mathrm{m} \mathrm{d}^{-1} \text {; assume that, } \\
\text { as in grazing fish, } 0.8 \% \text { of } P_{\mathrm{N}}=0.0002 \mathrm{~g} \mathrm{C} \mathrm{m} \mathrm{m}^{-2} \mathrm{~d}^{-1} \text { goes to zooplankton (i.e. reproduction) }\end{array}$ \\
\hline & $11 \rightarrow$ export & 0.0298 & The remainder of production $=0.0298 \mathrm{~g} \mathrm{C} \mathrm{m}^{-2} \mathrm{~d}^{-1}$ is exported from the system \\
\hline & $11 \rightarrow 18$ & 0.0623 & Since $\mathrm{AE}=70 \%, 30 \%$ of $\mathrm{C}=0.0623 \mathrm{~g} \mathrm{C} \mathrm{m}^{-2} \mathrm{~d}^{-1}$ is lost to column detritus \\
\hline & $R$ & 0.116 & See calculations for $11 \rightarrow 8$ \\
\hline \multirow[t]{4}{*}{$\begin{array}{l}\text { Planktuvorous fish } \\
\text { (Cpt 12) }\end{array}$} & $12 \rightarrow 8$ & 00003 & 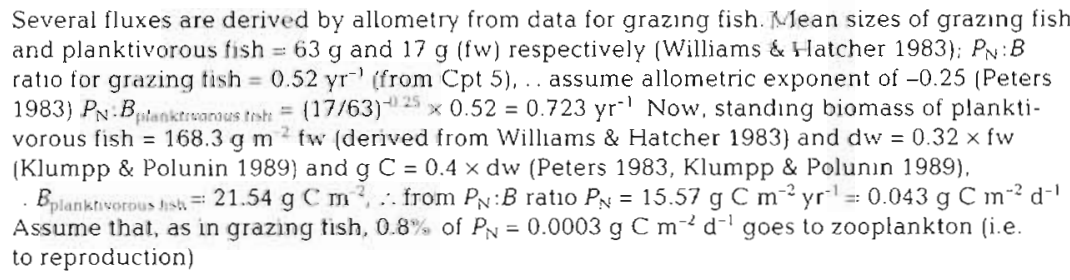 \\
\hline & $12 \rightarrow 11$ & 0.0427 & The remainder of production $=0.0427 \mathrm{~g} \mathrm{C} \mathrm{m}^{-2} \mathrm{~d}^{-1}$ is lost to piscivorous fish \\
\hline & $12 \rightarrow 18$ & 0.088 & $\begin{array}{l}C=\left(P_{\mathrm{N}}+R\right) / \mathrm{AE} \ldots \text { assuming } \mathrm{AE}=70 \%(8 \% \text { greater than for fish eating algae }) \\
C=(0.043+0.161) / 0.7=0.293 \mathrm{~g} \mathrm{C} \mathrm{m}^{-2} \mathrm{~d}^{-1} \text {, and assume that. as in grazıng fish, } 30 \% \text { of } \\
C=0.088 \mathrm{~g} \mathrm{C} \mathrm{m}^{-2} \mathrm{~d}^{-1} \text { is lost as faeces to column detntus }\end{array}$ \\
\hline & $R$ & 0.162 & $\begin{array}{l}\left.R_{\text {grazng fith }}=0.018 \mathrm{~g} \mathrm{C} \mathrm{m}^{-2} \mathrm{~d}^{-1}: B_{\text {grazang fish }}=3.277 \mathrm{~g} \mathrm{C} \mathrm{m}^{-2} \text { (see Cpts } 5 \text { and } 10\right) ; \ldots \text { by allometry } \\
\text { (Peters 1983), } R_{\text {planktwarous fish }}=(17 / 63)^{-0.25} \times(21.54 / 3.277) \times 0.018=0.162 \mathrm{~g} \mathrm{C} \mathrm{m}^{-2} \mathrm{~d}^{-1}\end{array}$ \\
\hline \multirow[t]{3}{*}{$\begin{array}{l}\text { Meiofauna } \\
\text { (Cpt 13) }\end{array}$} & $13 \rightarrow 9$ & 0.00005 & 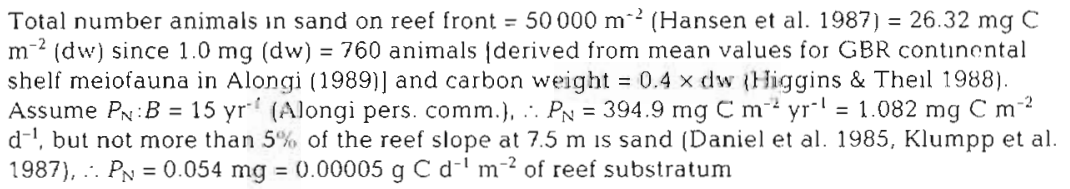 \\
\hline & $13 \rightarrow 19$ & 0.00207 & By difference \\
\hline & $R$ & 0.00018 & $\begin{array}{l}A E_{\text {merotauna }}=10 \% \text { (Alongi pers. comm). and } R=(C \times A E)-P_{N}=0.1 \times 0.0023-0.00005= \\
0.00018 \mathrm{~g} \mathrm{C} \mathrm{m}^{-2} \mathrm{~d}^{-1}\end{array}$ \\
\hline \multirow[t]{3}{*}{$\begin{array}{l}\text { Sedimentary } \\
\text { protozoa } \\
\text { (Cpt 14) }\end{array}$} & $14 \rightarrow 13$ & 0.0003 & 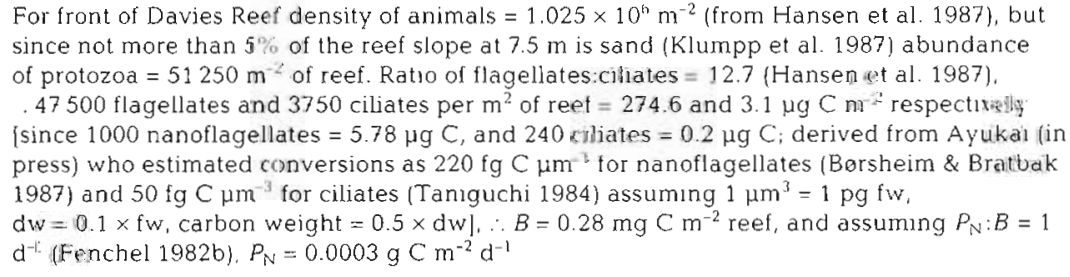 \\
\hline & $14 \rightarrow 19$ & 0.0005 & By difference \\
\hline & $R$ & 0.0005 & Assuming $\mathrm{AE}=60 \%$ (Fenchel 1982a), $R=(0.6 \times C)-P_{\mathrm{N}}=0.0005 \mathrm{~g} \mathrm{C} \mathrm{m}^{-2} \mathrm{~d}^{-1}$ \\
\hline
\end{tabular}


Appendix 1, continued

\begin{tabular}{|c|c|c|c|}
\hline Compartment & Flow & $\begin{array}{l}\text { Magnitude of flow } \\
\qquad\left(\mathrm{g} \mathrm{C} \mathrm{m}^{-2} \mathrm{~d}^{-1}\right)\end{array}$ & Source/derivation \\
\hline \multirow[t]{5}{*}{$\begin{array}{l}\text { Sedimentary } \\
\text { bacteria } \\
\text { (Cpt 15) }\end{array}$} & $15 \rightarrow 9$ & 0.001 & 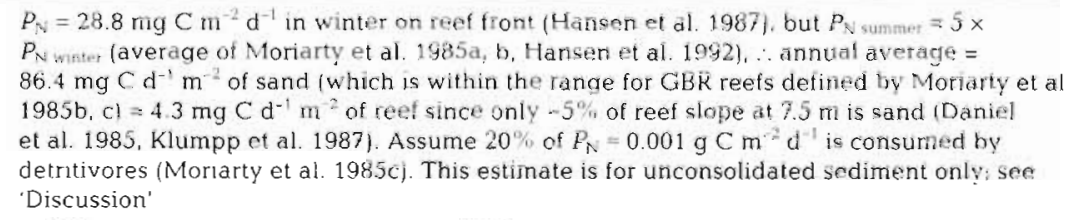 \\
\hline & $15 \rightarrow 13$ & 0.002 & $\begin{array}{l}\text { Average ratio of carbon biomass of bacteria consumed } \mathrm{d}^{-1} \text { by meiofauna: carbon biomass of } \\
\text { meiofauna }=1.8 \text { (Montagna } 1984)_{1} \therefore \text { bactena consumed }=26.32 \mathrm{mg} \mathrm{C} \mathrm{m}^{-2} \times 0.05 \% \times 1.8= \\
0.002 \mathrm{~g} \mathrm{C} \mathrm{m}^{-2} \mathrm{~d}^{-1}\end{array}$ \\
\hline & $15 \rightarrow 14$ & 0.0013 & Assume $30 \%$ of $P_{\mathrm{N}}=0.0013 \mathrm{~g} \mathrm{Cm} \cdot \mathrm{d}$ consumed by sedimentary protozoa (Moriarty et al. 1985) \\
\hline & $15 \rightarrow 1.9$ & 0.0084 & By difference \\
\hline & $R$ & 0.004 & $\begin{array}{l}\text { Average benthic community respiration }=18.3 .5 \mathrm{mg} \mathrm{C} \mathrm{m}^{-2} \mathrm{~d}^{-1} \text { (Hansen et al. 1992); assume } \\
70 \% \text { of this }=129.9 \mathrm{mg} \mathrm{C} \mathrm{m} \mathrm{m}^{-1} \text { is due to bacteria (Alongi pers. comm.). } \therefore \text { since average } \\
\text { production of benthic bacteria is } 139.3 \mathrm{mg} \mathrm{C} \mathrm{m}^{-2} \mathrm{~d}^{-1} \text { (Hansen et al. 1992), } P_{\mathrm{N}}: R=1.072 \text {, } \\
\therefore R=4.3 / 1.072=0.0040 \mathrm{~g} \mathrm{C} \mathrm{m}^{-2} \mathrm{~d}^{-1}\end{array}$ \\
\hline \multirow{5}{*}{$\begin{array}{l}\text { Water column } \\
\text { bacteria } \\
\text { (Cpt } 16\}\end{array}$} & Import & 142.8 & $\begin{array}{l}\text { Seasonal average of bacterial biomass on front of Davies Reef }=11.9 \mathrm{mg} \mathrm{C} \mathrm{m}^{-3} \text { (Ayukai in } \\
\left.\text { press) } \times \text { flow rate of } 12000 \mathrm{~m}^{3} \text { water } \mathrm{d}^{-1} \mathrm{~m}^{-1} \text { reef front (see Cpt } 1\right)=142.8 \mathrm{~g} \mathrm{C} \mathrm{m}^{-2} \mathrm{~d}^{-1}\end{array}$ \\
\hline & $16 \rightarrow 17$ & 0.0212 & $\begin{array}{l}\text { Assume } A E_{\text {columin protozod }}=0.6(\text { Fenchel 1982a }), \therefore C_{\text {column protozod }}=\left(P_{\mathrm{N}}+R\right) / 0.6=0.0212 \mathrm{~g} \mathrm{C} \\
\mathrm{m}^{-2} \mathrm{~d}^{-1}(\text { see } \mathrm{Cpt} 17)\end{array}$ \\
\hline & $16 \rightarrow 18$ & 0.032 & 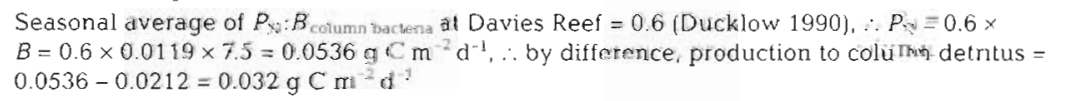 \\
\hline & Export & 142.9038 & By difference \\
\hline & $R$ & 0.050 & $\begin{array}{l}\text { Assume same } P_{\mathrm{N}}: R \text { ratio as for sedimentary bacteria }=1.072, \therefore R=0.0536 / 1.072=0.050 \mathrm{~g} \mathrm{C} \\
\mathrm{m}^{-2} \mathrm{~d}^{-1}\end{array}$ \\
\hline \multirow{6}{*}{$\begin{array}{l}\text { Water column } \\
\text { protozod and } \\
\text { other micro- } \\
\text { zooplankion } \\
\text { (Cpt 17) }\end{array}$} & Import & 7.176 & $\begin{array}{l}\left.\text { Mean standing biomass (averaged over summer } / \text { winter) }=11.96 \mathrm{mg} \mathrm{m}^{-3} \text { (fw) (Ayukai } 1991\right)= \\
0.598 \mathrm{mg} \mathrm{C} \mathrm{m} \text { since } \mathrm{dw}=0.1 \times \mathrm{fw} \text { and carbon weight }=0.5 \times \mathrm{dw}(\text { derived from Taniguchi } \\
\left.\text { 1984). Flow rate }=12000 \mathrm{~m}^{3} \mathrm{~d} \mathrm{~d}^{-1} \mathrm{~m}^{-1} \text { reef front (see Cpt } 1\right]=7.176 \mathrm{~g} \mathrm{C} \mathrm{m}^{-2} \mathrm{~d}^{-1}\end{array}$ \\
\hline & $17 \rightarrow 7$ & 0.060 & $\begin{array}{l}\left.\text { Coral clearance rate }=36.72 \mathrm{~m}^{3} \mathrm{~d}^{-1} \mathrm{~m}^{-2} \text { of coral surtace (see } \mathrm{Cpt} 18\right)=99.8 \mathrm{~m}^{3} \mathrm{~d}^{-1} \mathrm{~m}^{-2} \text { of } \\
\text { reef given mean coral rugosity factor of } 4.94 \text { and cover of corals at } 7.5 \mathrm{~m}=55 \% \text { (see Cpt } 18 \text { ). } \\
\text { Thus total mtake }=99.8 \times 0.598=0.060 \mathrm{~g} \mathrm{C} \mathrm{m}^{-2} \mathrm{~d}^{-1}\end{array}$ \\
\hline & $17 \rightarrow 8$ & 0.002 & $\begin{array}{l}\text { Assume zooplankton consume protozod and microzooplankton at the same rate as phyto- } \\
\text { plankton, . proportional to the standing biomass of phytoplankton (see Roman et al. } 1990\}= \\
0.598 \times(0.029 / 8.1)=0.002 \mathrm{~g} \mathrm{C} \mathrm{m}^{-2} \mathrm{~d}^{-1}\end{array}$ \\
\hline & $17 \rightarrow 18$ & 0.0085 & $\begin{array}{l}\text { Assume } P_{\mathrm{N}}: B=1 \mathrm{~d}^{-1} \text { (Fenchel } 1982 \mathrm{~b} \text { ): } B=0.598 \mathrm{mg} \mathrm{C} \mathrm{m} \mathrm{m}^{-3} \times 7.5 \mathrm{~m} \mathrm{depth}=4.485 \mathrm{mg} \mathrm{C} \mathrm{m}^{-2} \\
\left.\therefore P_{\mathrm{N}}=4.485 \mathrm{mg} \mathrm{C} \mathrm{m} \mathrm{m}^{-2} \text {. Assume } \mathrm{AE}=0.6 \text { (Fenchel } 1982 \mathrm{a}\right) . \therefore C=\left(P_{\mathrm{N}}+R\right) / 0.6=0.0212 \mathrm{~g} \mathrm{C} \\
\mathrm{m}^{-2} \mathrm{~d}^{-1} \text {. Assume all metabolic waste to detritus, } \therefore C-P_{\mathrm{N}}-R=0.0085\end{array}$ \\
\hline & Export & 7.1185 & $\begin{array}{l}\text { By difference; export < import follows observations of Ayukal (1991, unpubl.) that } \\
\text { standing stocks decrease from reef front to reef flat }\end{array}$ \\
\hline & $R$ & 0.0082 & $\begin{array}{l}\text { Assume same } P_{\mathrm{N}}: R \text { ratio as for sedimentary protozoa }=0.55 \ldots R=0.0045 / 0.55=0.0082 \mathrm{gC} \\
m_{1}^{-2} \mathrm{~d}^{-1}\end{array}$ \\
\hline \multirow[t]{2}{*}{$\begin{array}{l}\text { Water column } \\
\text { detritus } \\
\text { (Cpt 18) }\end{array}$} & Import & 795.6 & 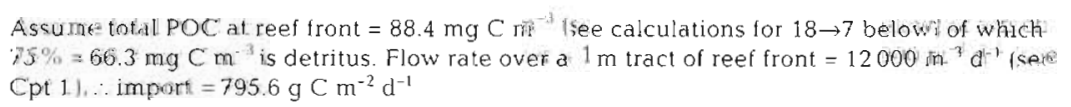 \\
\hline & $18 \rightarrow 7$ & 6.6 & 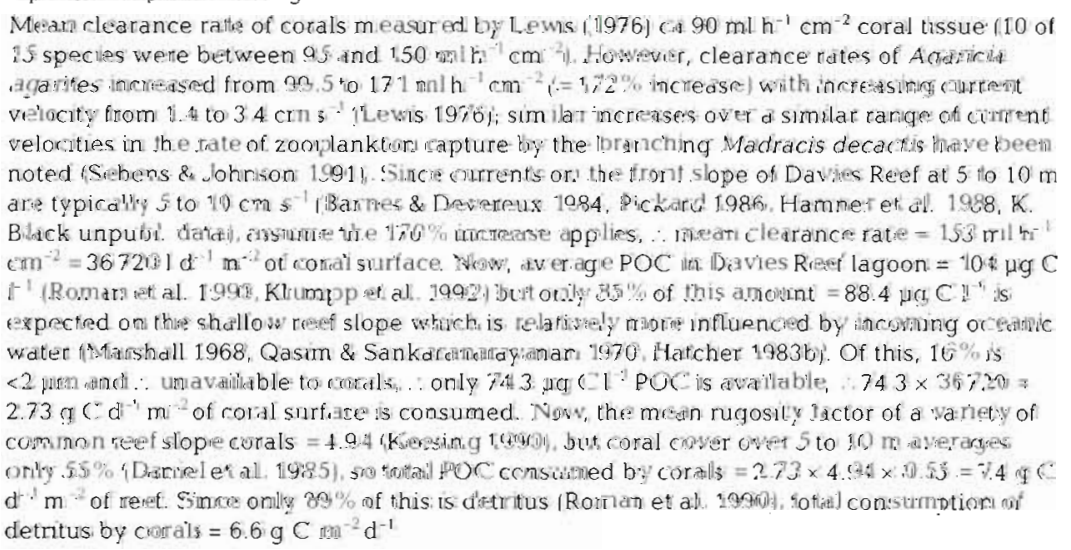 \\
\hline
\end{tabular}


Appendix 1, continued

\begin{tabular}{|c|c|c|c|}
\hline Compartment & Flow & $\begin{array}{l}\text { Magnitude of flow } \\
\qquad\left(\mathrm{g} \mathrm{C} \mathrm{m} \mathrm{Cl}^{-2} \mathrm{~d}^{1}\right)\end{array}$ & Source/derivation \\
\hline \multirow[t]{5}{*}{$\begin{array}{l}\text { Water column } \\
\text { detritus } \\
\text { (Cpt 18) }\end{array}$} & $18 \rightarrow 8$ & 0.052 & 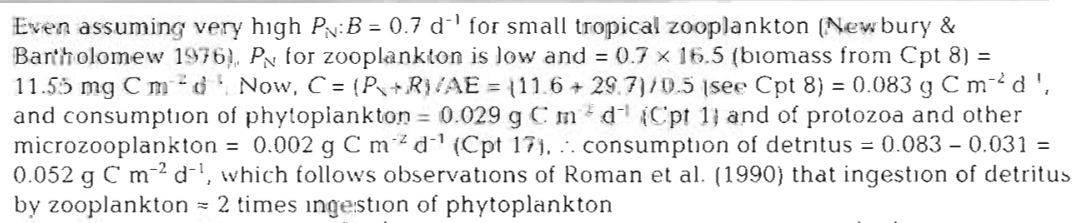 \\
\hline & $18 \rightarrow 12$ & 0.073 & 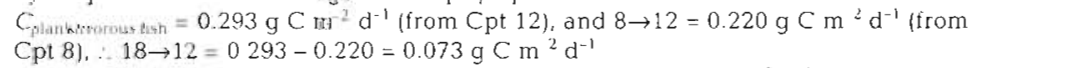 \\
\hline & $18 \rightarrow 16$ & 0.207 & $\mathrm{AE}_{\text {barterto }}=50 \%$ (Ducklow 1983) $\therefore C=\left(P_{\mathrm{N}}+R\right) / 0.5=0.207 \mathrm{~g} \mathrm{C} \mathrm{m}^{-2} \mathrm{~d}^{-1}$ (from Cpt 16) \\
\hline & $18 \rightarrow 19$ & 2.75968 & By difference (balancing values for Cpt 19) \\
\hline & Export & 793.97712 & By difference (balancing values for Cpt 18) \\
\hline \multirow{3}{*}{$\begin{array}{l}\text { Sedimitary } \\
\text { detritus } \\
\text { (Cpt 19) }\end{array}$} & $19 \rightarrow 6$ & 0.01 & $\begin{array}{l}\text { Assume that in grazıng 'micro' turt algae } 20 \% \text { of } \mathrm{C} \text { intake of invertivorous grazers is } \\
\text { detritus, } \ldots=0.2 \times(0.04 / 0.8)=0.01 \mathrm{~g} \mathrm{C} \mathrm{m}^{-2} \mathrm{~d}^{-1} \text { (see } \mathrm{Cpt} 3 \text { ) }\end{array}$ \\
\hline & $19 \rightarrow 9$ & 6.50695 & $\begin{array}{l}\text { Total consumption by detritivorous/carnivorous invertebrates }=6.517 \mathrm{~g} \mathrm{C} \mathrm{m}^{-2} \mathrm{~d}^{-1} \\
\text { (see Cpt } 9 \text { ). Of this } 0.009,0.00005 \text {, and } 0.001 \mathrm{~g} \mathrm{C} \mathrm{m}^{-2} \mathrm{~d}^{-1} \text { comes from grazing invertebrates, } \\
\text { meiofauna, and sedimentary bacteria respectively }(\mathrm{Cpts} 6,13 \text { and } 15) \ldots \text { by difference } \\
19 \rightarrow 9=6.50695 \mathrm{~g} \mathrm{C} \mathrm{m}^{-2} \mathrm{~d}^{-1}\end{array}$ \\
\hline & $19 \rightarrow 15$ & 0.0167 & $\mathrm{AE}_{\text {bacteria }}=50 \%$ (Ducklow 1983) $\ldots C=\left(P_{\mathrm{N}}+R\right) / 0.5=0.0167 \mathrm{~g} \mathrm{C} \mathrm{m}^{-2} \mathrm{~d}^{-1}($ from Cpt 15) \\
\hline
\end{tabular}

Appendix 2. Summary of derivations of estimates of carbon flux $\lg \mathrm{m}^{-2}$ $\mathrm{d}^{-1}$ ) on the front slope of Davies Reef (depth 5 to $10 \mathrm{~m}$. mean depth $7.5 \mathrm{~m}$ ) for the algae-dominated state after destruction of corals by crown-ofthorns startish Acanthaster planci. Two scenarios are given: (1) coral cover is reduced to $2 \%$ (Keesing 1990 ) but grizers do not respond to the increased availability of algae (e.g. Williams 1986) so the increase in production of algal carbon flows to water column detritus, and (2) coral cover is reduced to $2 \%$ and grazers respond to increased availability of algae (e.g. Robertson 1991) so that losses of alqal carbon to grazers and losses to detritus are in the same proportion (ca 1:1) as occurs in the coraldominated state (see 'Discussion'). In these models it was assumed that production of the epilithic algal community (EAC) (corallıne algae (COA) + turf algae] per unt area of algae-covered substratum on starfish-affected reefs is identical to that on coral-dominated reefs isee 'Methods'), although the amount of algae-covered substratum is clearly greater on starfish-affected reefs. Flows are calculated assuming steady state for each compartment and for the system as a whole. Flows $X \rightarrow Y$ indicate flows from compartment $X$ to compartment $Y$. The number of decimal places does not indicate the precision of the estimates but is required in some places to balance flows. Note that exogenous imports consider only water that eventually flows over the reef but not that which flows along the front and around the reef to exit downstream (see 'Methods' for details). Abbreviations as for Appendix 1

Scenario 1 . Coral cover is $2 \%$, excess in production of algal carbon flows to detrital pathways. Fluxes for corals [i.e. ingestion, losses to column detritus, reproduction (i.e. the flux from coral $\rightarrow$ zooplankton) and respiration] and zooxanthellae (production and respiration) were scaled down from the coral-dominated configuration by a factor of $55 / 2=27.5$. Compartments for phytoplankton, zooplankton, water column detritus, and water column protozoa were balanced by adjusting exports. Assuming dead coral skeletons are colonised by EAC (turf algae + COA), production and respiration of these groups was scaled up by a factor of $(41 \%+53 \%) / 41 \%=2.29$ to account for their increase in cover. In this scenario, there is no increase in grazer activity so the increase in net algal production is lost as detritus. Magnitudes of flows are summarised.

Scenario 2 . Coral cover is $2 \%$, fate of algal production is $50 \%$ lost to grazers and $50 \%$ exported as defritus. Fluxes for corals (heterotrophic) and zooxanthellae, and gross production and respration of other hoterotrophs were determined as described for Scenario 1. However, net production was apportioned among flows to grazers and detritus in the same: proportion as in the coral-dominated state. This carbon was then allowed to flow through the system in such a way as to maintain the characteristic assimilation efficiencies, $P: R$ ratios, etc. of each trophic compartulent. Magnitudes of flows are summarised

\begin{tabular}{|c|c|c|c|}
\hline Compartment & Flow & $\begin{array}{c}\text { Scenano } 1 \\
\text { magnitude of flow } \\
\left(\mathrm{g} \mathrm{C} \mathrm{m}^{-2} \mathrm{~d}^{-1}\right)\end{array}$ & $\begin{array}{l}\text { Scenario } 2 \\
\text { magnitude of flow } \\
\left(\mathrm{g} \mathrm{C} \mathrm{m}^{-2} \mathrm{~d}^{-1}\right)\end{array}$ \\
\hline $\begin{array}{l}\text { Phytoplankton } \\
\text { (Cpt 1) }\end{array}$ & $\begin{array}{l}\text { Imported } \\
1 \rightarrow 7 \\
1 \rightarrow 8 \\
R \\
\text { Exported }\end{array}$ & $\begin{array}{l}97.20 \\
0.027 \\
0.029 \\
0 \\
97.144\end{array}$ & $\begin{array}{c}97.20 \\
0.027 \\
0.029 \\
0 \\
97.144\end{array}$ \\
\hline $\begin{array}{l}\text { Corals: } \\
\text { zooxanthellae } \\
\text { (Cpt 2) }\end{array}$ & $\begin{array}{l}P_{G} \\
2 \rightarrow 7 \\
R\end{array}$ & $\begin{array}{l}0.54 \\
0.458 \\
0.082\end{array}$ & $\begin{array}{l}0.54 \\
0.458 \\
0.082\end{array}$ \\
\hline $\begin{array}{l}\text { Turf algae } \\
\text { (Cpt 3) }\end{array}$ & $\begin{array}{l}P_{G} \\
3-\rightarrow 5 \\
3-\rightarrow 6 \\
3 \rightarrow 18 \\
R\end{array}$ & $\begin{array}{l}1.49 \\
0.14 \\
0.04 \\
0.645 \\
0.665\end{array}$ & $\begin{array}{l}1.49 \\
0.33 \\
0.083 \\
0.412 \\
0.665\end{array}$ \\
\hline $\begin{array}{l}\mathrm{COA} \\
(\mathrm{Cpt} 4)\end{array}$ & $\begin{array}{l}P_{G} \\
4 \rightarrow 5 \\
4 \rightarrow 18 \\
R\end{array}$ & $\begin{array}{l}0.986 \\
0.12 \\
0.407 \\
0.459\end{array}$ & $\begin{array}{l}0.986 \\
0.264 \\
0.263 \\
0.459\end{array}$ \\
\hline $\begin{array}{l}\text { Grazıng fish } \\
\text { (Cpt 5) }\end{array}$ & $\begin{array}{l}5 \rightarrow 8 \\
5 \rightarrow 11 \\
5 \rightarrow 18 \\
R\end{array}$ & $\begin{array}{l}0.001 \\
0.161 \\
0.11 \\
0.018\end{array}$ & $\begin{array}{l}0.002 \\
0.347 \\
0.236 \\
0.039\end{array}$ \\
\hline $\begin{array}{l}\text { Invertebrate } \\
\text { grazers } \\
\text { (Cpt 6) }\end{array}$ & $\begin{array}{l}6 \rightarrow 9 \\
6 \rightarrow 10 \\
6 \rightarrow 18 \\
R\end{array}$ & $\begin{array}{l}0.009 \\
0.01 \\
0.025 \\
0.006\end{array}$ & $\begin{array}{l}0.017 \\
0.019 \\
0.046 \\
0.011\end{array}$ \\
\hline $\begin{array}{l}\text { Corals: } \\
\text { heterotrophic } \\
\text { (Cpt 7) }\end{array}$ & $\begin{array}{l}7 \rightarrow 8 \\
7 \rightarrow 18 \\
R\end{array}$ & $\begin{array}{l}0.096 \\
0.27 \\
0.41\end{array}$ & $\begin{array}{l}0.096 \\
0.270 \\
0.410\end{array}$ \\
\hline $\begin{array}{l}\text { Zooplankton } \\
\text { (Cpt 8) }\end{array}$ & $\begin{array}{l}\text { Imported } \\
8 \rightarrow 5 \\
8 \rightarrow 7 \\
8 \rightarrow 12 \\
8 \rightarrow 18 \\
\text { Exported } \\
R\end{array}$ & $\begin{array}{c}26.4 \\
0.03 \\
0.049 \\
0.22 \\
0.043 \\
26.2089 \\
0.03\end{array}$ & $\begin{array}{c}26.4 \\
0.03 \\
0.049 \\
0.220 \\
0.043 \\
26.2103 \\
0.03\end{array}$ \\
\hline
\end{tabular}

(Appendix 2 continued on next page) 
Appendix 2, continued

\begin{tabular}{|c|c|c|c|}
\hline Compartment & ma & $\begin{array}{l}\text { Scenario } 1 \\
\text { magnutude of flow } \\
\text { (g }\left(\mathrm{Cm}^{-2} \mathrm{~d}^{-1}\right)\end{array}$ & $\begin{array}{l}\text { Scenario } 2 \\
\text { magnitude of flow } \\
\left(\mathrm{g} \mathrm{C} \mathrm{m} \mathrm{m}^{-2} \mathrm{~d}^{-1}\right)\end{array}$ \\
\hline $\begin{array}{l}\text { Carnivorous and } \\
\text { detritivorous } \\
\text { invertebrates } \\
\text { (Cpt 9) }\end{array}$ & $\begin{array}{l}9 \rightarrow 10 \\
9 \rightarrow 19 \\
R\end{array}$ & $\begin{array}{l}0.024 \\
3.763 \\
2.73\end{array}$ & $\begin{array}{l}0.024 \\
3.770 \\
2.736\end{array}$ \\
\hline $\begin{array}{l}\text { Invertivorous fish } \\
\text { (Cpt 10) }\end{array}$ & $\begin{array}{l}10 \rightarrow 8 \\
10 \rightarrow 11 \\
10 \rightarrow 18 \\
R\end{array}$ & $\begin{array}{l}0.0004 \\
0.0046 \\
0.010 \\
0.019\end{array}$ & $\begin{array}{l}0.0005 \\
0.0059 \\
0.0126 \\
0.024\end{array}$ \\
\hline $\begin{array}{l}\text { Piscivorous fish } \\
\text { (Cpt 11) }\end{array}$ & $\begin{array}{l}11 \rightarrow 8 \\
11 \rightarrow 18 \\
11 \rightarrow \text { export } \\
R\end{array}$ & $\begin{array}{l}0.0002 \\
0.0623 \\
0.0298 \\
0.116\end{array}$ & $\begin{array}{l}0.0005 \\
0.1187 \\
0.0574 \\
0.219\end{array}$ \\
\hline $\begin{array}{l}\text { Planktivorous fish } \\
\text { (Cpt 12) }\end{array}$ & $\begin{array}{l}12 \rightarrow 8 \\
12 \rightarrow 11 \\
12 \rightarrow 18 \\
R\end{array}$ & $\begin{array}{l}0.0003 \\
0.0427 \\
0.088 \\
0.162\end{array}$ & $\begin{array}{l}0.0003 \\
0.0427 \\
0.088 \\
0.162\end{array}$ \\
\hline $\begin{array}{l}\text { Meiofauna } \\
\text { (Cpt } 13)\end{array}$ & $\begin{array}{l}13 \rightarrow 9 \\
13 \rightarrow 19 \\
R\end{array}$ & $\begin{array}{l}0.00005 \\
0.00207 \\
0.00018\end{array}$ & $\begin{array}{l}0.00005 \\
0.00207 \\
0.00018\end{array}$ \\
\hline $\begin{array}{l}\text { Sedimentary } \\
\text { protozoa } \\
\text { (Cpt 14) }\end{array}$ & $\begin{array}{l}14 \rightarrow 13 \\
14 \rightarrow 19 \\
R\end{array}$ & $\begin{array}{l}0.0003 \\
0.0005 \\
0.0005\end{array}$ & $\begin{array}{l}0.0003 \\
0.0005 \\
0.0005\end{array}$ \\
\hline $\begin{array}{l}\text { Sedimentary } \\
\text { bacterla } \\
\text { (Cpt } 15)\end{array}$ & $\begin{array}{l}15 \rightarrow 9 \\
15 \rightarrow 13 \\
15 \rightarrow 14 \\
15 \rightarrow 19 \\
R\end{array}$ & $\begin{array}{l}0.001 \\
0.002 \\
0.0013 \\
0.0084 \\
0.004\end{array}$ & $\begin{array}{l}0.001 \\
0.002 \\
0.0013 \\
0.0084 \\
0.004\end{array}$ \\
\hline $\begin{array}{l}\text { Water column } \\
\text { bacteria } \\
\text { (Cpt 16) }\end{array}$ & $\begin{array}{l}\text { Import } \\
16 \rightarrow 17 \\
16 \rightarrow 18 \\
\text { Export } \\
R\end{array}$ & $\begin{array}{c}142.8 \\
0.0212 \\
0.032 \\
142.9038 \\
0.050\end{array}$ & $\begin{array}{c}142.8 \\
0.0212 \\
0.032 \\
142.9038 \\
0.050\end{array}$ \\
\hline $\begin{array}{l}\text { Water column } \\
\text { protozoa and } \\
\text { other mucro- } \\
\text { zooplankton } \\
\text { (Cpt 17) }\end{array}$ & $\begin{array}{l}\text { Import } \\
17 \rightarrow 7 \\
17 \rightarrow 8 \\
17 \rightarrow 18 \\
\text { Export } \\
R\end{array}$ & $\begin{array}{l}7.176 \\
0.002 \\
0.002 \\
0.0085 \\
7.1765 \\
0.0082\end{array}$ & $\begin{array}{l}7.176 \\
0.002 \\
0.002 \\
0.0085 \\
7.1765 \\
0.0082\end{array}$ \\
\hline $\begin{array}{l}\text { Water column } \\
\text { detritus } \\
\text { (Cpt 18) }\end{array}$ & $\begin{array}{l}\text { Import } \\
18 \rightarrow 7 \\
18 \rightarrow 8 \\
18 \rightarrow 12 \\
18 \rightarrow 16 \\
18 \rightarrow 19 \\
\text { Export }\end{array}$ & $\begin{array}{c}795.6 \\
0.24 \\
0.052 \\
0.073 \\
0.207 \\
2.75968 \\
793.96912\end{array}$ & $\begin{array}{c}795.6 \\
0.24 \\
0.052 \\
0.073 \\
0.207 \\
2.75768 \\
793.80012\end{array}$ \\
\hline $\begin{array}{l}\text { Sedimentary } \\
\text { detritus } \\
\text { (Cpt 19) }\end{array}$ & $\begin{array}{l}19 \rightarrow 6 \\
19 \rightarrow 9 \\
19 \rightarrow 15\end{array}$ & $\begin{array}{l}0.01 \\
6.50695 \\
0.0167\end{array}$ & $\begin{array}{l}0.01 \\
6.51195 \\
0.0167\end{array}$ \\
\hline
\end{tabular}

\section{LITERATURE CITED}

Adey WH (1983) The microcosm: a new tool for reef research. Coral Reefs 1:193-201

Alldredge AL, King JM (1977) Distribution, abundance, and substrate preferences of demersal reef zooplankton at Lizard Island Lagoon, Great Barrier Reef. Mar Biol 41: $317-333$

Alongi DM (1989) Benthic processes across mixed terrigenouscarbonate sedimentary facies on the central Great Barrier Reef continental shelf. Cont Shelf Res 9:629-663
Atkinson MJ, Grigg RW (1984) Model of a coral reef ecosystem. II. Gross and net benthic primary production at French Frigate Shoals, Hawaii. Coral Reefs 3:13-22

Ayukai I (1991) Standing stock of microzooplankton on coral reefs: a preliminary study. J Plankton Res 13:895-899

Ayukai $T$ (in press) Retention of phytoplankton and planktonic microbes on coral reefs in the Great Barrier Reef, Australia. Coral Reefs

Baird D, Ulanowicz RE (1989) The seasonal dynamics of the Chesapeake Bay ecosystem. Ecol Monogr 59:329-364

Bak RPM (1990) Patterns of echinoid bioerosion in two Pacific coral reef lagoons. Mar Ecol Prog Ser 66:267-272

Banse K (1977) Determining the carbon-to-chlorophyll ratio of natural phytoplankton. Mar Biol 41:199-212

Barnes DJ (1988) Seasonality in community productivity and calcification at Davies Reef, central Great Barrier Reef. Proc 6th Int Symp Coral Reef 2:521-527

Barnes DJ, Devereux MJ (1984) Productivity and calcification on a coral reef: a survey using $\mathrm{pH}$ and oxygen electrode techniques. J exp mar Biol Ecol 79:213-231

Birkeland C, Lucas JS (1990) Acanthaster planci: major management problem of coral reefs. CRC Press, Boca Raton

Borsheim KY, Bratbak G (1987) Cell volume to cell carbon conversion factors for a bacterivorous Moras sp. enriched from seawater. Mar Ecol Prog Ser 36:171-175

Brown BE (1987) Worldwide death of corals: natural cyclic events or man-made pollution? Mar Pollut Bull 18:9-13

Carpenter RC (1985) Relationships between primary production and i.radiance in coral reef algal communities. Limnol Oceanogr 30:784-793

Carpenter RC (1990) Mass mortality of Diadema antillarum I. Long-term effects on sea urchin population-dynamics and coral reef algal communities. Mar Biol 104:67-77

Chapin FS, Schulze ED, Mooney HA (1992) Biodiversity and ecosystem processes. Trends Ecol Evol 7: 107-108

Chardy P. Clavier J (1988) An attempt to estimate the carbon budget for the south west lagoon of New Caledonia. Proc 6th Int Symp Coral Reefs 2:541-54.6

Coffroth MA (1990) Mucous sheet formation on poritid corals: an evaluation of coral mucus as a nutrient source on reefs Mar Biol 105:39-49

Cooksey K, Cooksey B (1972) Turnover of photosynthetically fixed carbon in reef corals. Mar Biol 15:289-292

Crossland CJ (1980) Release of photosynthetically-derived organic carbon from a hermatypic coral, Acropora cf. acuminata. In: Schwemmler W, Schenk HEA (eds) Endocytobiology, endosymbiosis and cell biology. A synthesis of recent research. Proceedings of the International Colloquium on Endosymbiosis and Cell Research, Tübingen, Germany. de Gruyter, Berlin, p 163-172

Crossland CJ, Barnes DJ, Cox T, Devereux M (1980) Compartmentation and turnover of organic carbon in the staghorn coral Acropora formosa. Mar Biol 59:181-187

Daniel PA, Johnson DB, DeVantier LM, Barnes GR (1985) Studies in the assessment of coral reef ecosystems. 7 Davies Reef. In: Bradbury RH, Reichelt RE (eds) Studies in the assessment of coral reef ecosystems. AIMS, Cape Ferguson, Queensland, Australia, p 1-39

Davies PS (1984) Carbon budgets and vertical zonation of Atlantic reef corals. Proc 3rd Int Symp Coral Reefs 1 . 391-395

D'Elia CF, Wiebe WJ (1990) Biogeochemical nutrient cycles in coral-reef ecosystems. In: Dubinsky $Z$ (ed) Ecosystems of the world 25: coral reefs. Elsevier, Amsterdam, p 49-74

Doherty PJ (1980) Biological and physical constraints on the populations of 2 sympatric territorial damselfishes on the southern GBR. PhD dissertation, University of Sydney 
Doherty PJ (1983) Diel, lunar and seasonal rhythms in the reproduction of two tropical damselfishes: Pomacentrus flavicauda and $P$. ward. Mar Biol 75:215-224

Doherty PJ, Williams DMcB (1989) The replenishment of coral reef fish populations. Oceanogr mar Biol A Rev 26: $487-551$

Done I (1992a) Constancy and change in some Great Barrier Reet coral communities: 1980-1990. Am Zool 32:655-662

Done TJ (1992b) Effects of tropical cyclone waves on ecological and geomorphological structures on the Great Barrier Reef. Cont Shelf Res 12:859-872

Done TJ (1992C) Phase shifts in coral reef communutues and their ecological significance. Hydrobiologia 247:121-132

Done TJ, Ogden JC, Wiebe WJ (in press) Biodiversity and ecosystem function of coral reefs. In: Mooney HA (ed) Biodiversity and ecosystem function: a global perspective. Wiley Interscience

Ducklow HT (1983) The production and fate of bacteria in the oceans. Biosci 33:494-501

Ducklow HT (1990) The biomass, production and fate of bacteria in coral reefs. In: Dubinsky Z (ed) Ecosystems of the world 25: coral reefs. Elsevier, Amsterdam, p 265-289

Edmunds PJ, Davies PS (1986) An energy budget for Porites porites (Scleractinia). Mar Biol 92:339-347

Erez $J(1990)$ On the importance of food sources in coral-reef ecosystems. In: Dubinsky $Z$ (ed) Ecosystems of the world 25: coral reefs. Elsevier, Amsterdam, p 411-418

Fenchel T (1982a) Ecology of heterotrophic microflagellates. II. Bioenergetics and growth. Mar Ecol Prog Ser 8:225-231

Fenchel T (1982b) Ecology of heterotrophic microflagellates. IV Quantitative occurrence and importance as bacterial consumers. Mar Ecol Prog Ser 9:35-42

Field JG, Moloney CL. Atwood CG (1989b) Network analysis of simulated succession after an upwelling event. In: Wulff F. Field JG, Mann KH (eds) Network analysis in marine ecology. Coastal and estuarine studies 32. Springer Verlag, Berlin, p 132-158

Field JG, Wulff F, Mann KH (1989a) The need to analyze ecological networks. In: Wulff $F$, Field JG, Mann KH (eds) Network analysis in marine ecology. Coastal and estuarine studies 32. Springer Verlag, Berlin, p 3-12

Furnas MJ, Mitchell AW, Gilmartin M, Revelante N (1990) Phytoplankton biomass and primary production in semienclosed reef lagoons of the central Great Barrier Reef, Australia. Coral Reefs 9:1-10

Glynn PW (1993) Coral reef bleaching: ecological perspectives. Coral Reefs 12:1-17

Glynn PW, Colgan MW (1992) Sporadic disturbances in fluctuating coral reef environments: El Nino and coral reef development in the eastern Pacific. Am Zool 32:707-718

Gottfried M, Roman MR (1983) Ingestion and incorporation of coral-mucus detritus by reef zooplankton. Mar Biol 72 : 211-218

Grigg RW, Polovina JJ, Atkinson MJ (1984) Model of a coral reef ecosystem. III. Resource limitation, community regulation, fisheries yield and resource management. Coral Reefs 3:23-27

Hackney JM, Carpenter RC, Adey WH (1989) Characteristic adaptations to grazing among algal turfs on a Caribbean coral reef. Phycologia 28:109-119

Hamner WM, Haur IR (1981) Effect of island mass: water flow and plankton pattern around a reef in the Great Barrier Reef lagoon, Australia. Limnol Oceanogr 26:1084-1102

Hamner WM, Jones MS, Carleton JH, Hauri IR, Williams DMcB (1988) Zooplankton, planktivorous fish, and water currents on a windward reef face: Great Barrier Reef, Australia. Bull mar Sci 42:459-479
Hamner WM, Wolanski E (1988) Hydrodynamic forcing functions and biological processes on coral reefs: a status review. Proc 6th Int Symp Coral Reefs 1:103-113

Hansen JA, Alongi DM, Moriarty DJW, Pollard PC (1987) The dynamics of benthic microbial communities at Davies Reef, central Great Barrier Reef. Coral Reefs 6:63-70

Hansen JA, Klumpp DW, Alongi DM, Dayton PK, Riddle MJ (1992) Detrital pathways in a coral reef lagoon. II. Detritus deposition, benthıc microbial biomass and production. Mar Biol 113:363-372

Hatcher BG (1983a) Grazing in coral reef ecosystems. In Barnes DJ (ed) Perspectives on coral reefs. B Clouston, Manuka, ACT, p 164-169

Hatcher BG (1983b) The role of detritus in the metabolism and secondary production of coral reef ecosystems. In: Baker JT, Carter RM, Sammarco PW, Stark KP (eds) Proceedings of the Great Barrier Reef Conference. James Cook University Press, Townsville, p 317-325

Hatcher BG (1990) Coral reef primary productivity: a hierarchy of pattern and process. Trends Ecol Evol 5:149-155

Hatcher BG, Johannes RE, Robertson AI (1989) Review of research relevant to the conservation of shallow water tropical marine ecosystems. Oceanogr mar Biol A Rev 27 $337-4.14$

Hay ME (1991) Fish-seaweed interactions on coral reefs: effects of herbivorous fishes and adaptations of their prey. In: Sale PF (ed) The ecology of fishes on coral reefs. Academic Press, San Diego, p 96-119

Higgins RP. Thiel H (eds) (1988) lntroduction to the study of meiofauna. Smithsonian Inst Press, Washington

Hughes TP (1989) Community structure and diversity of coral reefs: the role of history. Ecology 70:275-279

Hughes TP (1994) Catastrophes, phase shifts, and large scale degradation of a Caribbean coral reef. Science 265: $1547-1551$

Hutchings PA (1978) Non-colonial cryptofauna. In: Stoddart DR, Johannes RE (eds) Coral reefs: research methods UNESCO, Paris, p 251-261

Johannes RE, Coles SL, Kuenzel NT (1970) The role of zooplankton in the nutrition of some scleractinian corals Limnol Oceanogr 15:579-586

Johannes RE, Tepley L (1974) Examination of feeding of the reef coral Porites lobata in situ using time lapse photography. Proc 2nd Int Symp Coral Reefs 2:127-131

Johnson CR (ed) (1992) Crown-of-thorns starfish on the Great Barrier Reef: reproduction, recruitment and hydrodynamics. CSIRO, Melbourne

Johnson CR, Muir DG, Reysenbach AL(1991a) Characteristic bacteria associated with surfaces of coralline algae: a hypothesis for bacterial induction of marine invertebrate larvae. Mar Ecol Prog Ser 74:281-294

Johnson CR, Sutton DC, Olson RR, Giddins R (1991b) Settlement of crown-of-thorns starfish: role of bacteria on surfaces of coralline algae and a hypothesis for deepwater recruitment. Mar Ecol Prog Ser 74:143-162

Jokiel PL, Morrissey JI (1986) Influence of size on primary production in the reef coral Pocillopora damicornis and the macroalga Acanthophora spicifera. Mar Biol 91:15-26

Jorgensen CB (1955) Quantitative aspects of filter feeding in invertebrates. Biol Rev 30:391-454

Kay JJ, Graham LA, Ulanowicz RE (1989) A detailed guide to network analysis. In: Wulff $F$, Field JG, Mann KH (eds) Network analysis in marine ecology. Coastal and estuarine studies 32. Springer Verlag, Berlin, p 15-61

Keesing JK (1990) Feeding biology of the crown-of-thorns starfish, Acanthaster planci (Linnaeus). PhD dissertation, James Cook University of North Queensland, Townsville 
Kinsey DW (1985) Metabolism, calcification and carbon production. I. System level studies. Proc 5th Int Coral Reefs Congr 4:505-526

Klumpp DW, Bayne BL, Hawkins AJS (1992) Nutrition of the giant clam Tridacna gigas (L.). I. Contribution of filter feeding and photosynthates to respiration and growth. $\mathrm{J}$ exp mar Biol Ecol 155:105-122

Klumpp DW, McKinnon AD (1989) Temporal and spatial patterns in primary production of a coral-reef epilithic algal community. J exp mar Biol Eco 131:1-22

Klumpp DW, McKinnon AD (1992) Community structure, biomass and productivity of epilithic algal communities on the GBR: dynamics at different spatial scales. Mar Ecol Prog Ser 86:77-89

Klumpp DW, McKinnon AD, Daniel P (1987) Damselfish teritories: zones of high productivity on coral reefs. Mar Ecol Prog Ser 40:41-51

Klumpp DW, McKinnon AD, Mundy CN (1988) Motile cryptofauna of a coral reef: abundance, distribution and trophic potential. Mar Ecol Prog Ser 45:95-108

Klumpp DW, Polunin NVC (1989) Partitioning among grazers of food resources within damselfish territories on a coral reef. J exp mar Biol Ecol 131:1-22

Klumpp DW, Polunin NVC (1990) Algal production, grazers and habitat partitioning on a coral reef: positive correlation between grazing rate and food availability. In: Barnes M, Gibson R (eds) Trophic relationship in the marine environment. Proc 24 th Eur mar biol Symp, Aberdeen University Press, Aberdeen, p 372-388

Klumpp DW, Pulfrich A (1989) Trophic significance of herbivorous macroinvertebrates on the central GBR. Coral Reefs 8:135-144

Knowlton $N$ (1992) Thresholds and multiple stable states in coral reef community dynamics. Am Zool 32:674-682

Knowiton N, Lang JC, Keller BD (1990) Case study of natural population collapse: post-hurricane predation on Jamaican staghorn corals. Smithson Contr mar Sci 31:1-25

Lewis JB (1976) Experimental tests of suspension feeding in Atlantic reef corals. Mar Biol 36:147-150

Littler MM, Littler DS, Lapointe BE (1993) Modification of tropical reef community structure due to cultural eutrophication: the southwest coast of Martinique. Proc 7 th Int Symp Coral Reefs 1:335-343

Marshall N (1968) Observations on organic aggregates in the vicinity of coral reefs. Mar Biol 2:50-53

McCloskey LR, Muscatine L (1984) Production and respiration in the Red Sea coral Stylophora pistillata as a function of depth. Proc R Soc Lond B 222:215-230

Montagna PA (1984) In situ measurement of meiobenthic grazing rates on sediment bacterla and edaphic diatoms. Mar Ecol Prog Ser 18:119-130

Moran PJ (1986) The Acanthaster phenomenon. Oceanogr mar Biol A Rev 24:379-480

Moran PJ, De'ath G, Baker VJ, Bass DK, Chnstie CA, Miller IR, Miller-Smith BA, Thompson AA (1992) Pattern of outbreaks of crown-of-thorns starfish (Acanthaster planci L.) along the Great Barrier Reef since 1966. Aust J mar Freshwat Res 43:555-568

Moriarty DJW (1979) Biomass of suspended bacteria over coral reefs. Mar Biol 53:193-200

Moriarty DJW, Pollard PC, Alongi DM, Wilkinson CR, Gray JS (1985c) Bacterial productivity and trophic relationships with consumers on a coral reef (MECOR I). Proc 5th Int Coral Reefs Congr 3:457-462

Monarty DJW. Pollard PC Hunt WG (1985a) Temporal and spatial variation in bacterial production in the water column over a coral reef. Mar Biol 85:285-292
Moriarty DJW, Pollard PC. Hunt WG, Moriarty CM, Wassenberg TJ (1985b) Productivity of bacteria and microalgae and the effect of grazing by holothurians in sediments on a coral reef flat. Mar Biol 85:293-300

Muscatine $\mathrm{L}(1990)$ The role of symbiotic algae in carbon and energy flux in reef corals. In: Dubinsky $Z$ (ed) Ecosystems of the world 25: coral reefs. Elsevier, Amsterdam, p 75-87

Muscatine L, Falkowski P, Porter J, Dubinsky Z (1984) Fate of photosynthetically-fixed carbon in light and shadeadapted colonies of the symbiotic coral Stylophora pistillata. Proc R Soc Lond B 222:181-202

Muscatine L, Porter JW (1977) Reef corals: mutualistic symbioses adapted to nutrient-poor environments. BioSci 27 $454-460$

Newbury TK, Bartholomew EF (1976) Secondary production of microcopepods in the southern, eutrophic basin of Kaneohe Bay, Oahu, Hawaiian Islands. Pacif Sci 30: 373-384

Omori M, Ikeda T (1984) Methods in marine zooplankton ecology. Wiley, New York

Padilla DK (1989) Algal structural defenses: form and calcification in resistance to tropical limpets. Ecology 70 : $835-842$

Peters RH (1983) The ecological implications of body size. Cambridge University Press, New York

Pickard GL (1986) Effects of wind and tide on upper layer currents at Davies reef, Great Barier Reef, during MECOR (July/August 1984). Aust J mar Freshwat Res 37: $545-565$

Polovina JJ (1984) Model of a coral reef ecosystem. I. The ECOPATH model and its application to French Frigate Shoals. Coral Reefs 3:1-11

Polunin NVC (1988) Efficient uptake of algal production by a single resident herbivorous fish on the reef. J exp mar Biol Ecol 123:61-76

Polunin NVC, Klumpp DW (1989) Ecological correlates of foraging periodicity in herbivorous reef-fishes of the Coral Sea. J exp mar Biol Ecol 126:1-20

Porter JW (1974) Zooplankton feeding by the Caribbean reefbuilding coral Montastrea cavernosa. Proc 2nd Int Symp Coral Reefs 1:111-125

Porter JW (1976) Autotrophy, heterotrophy, and resource partitioning in Caribbean reef-building corals. Am Nat 110: $731-742$

Qasim SZ, Sankaranarayanan VN (1970) Production of particulate organic matter by the reef on Kavaratti atoll (Laccadives). Limnol Oceanogr 15:574-578

Riddle MJ, Alongi DM, Dayton PK, Hansen JA, Klumpp DW (1990) Detrital pathways in a coral reef lagoon. I. Macrofaunal biomass and estimates of production. Mar Biol 104: $109-118$

Robertson DR (1991) Increases in surgeon fish populations after mass mortality of the sea urchin Diadema antllarum in Panama indicates food limitation. Mar Biol 111:437-444

Roman MR, Furnas MJ, Mullin MM (1990) Zooplankton abundance and grazing at Davies Reef, Great Barrier Reef, Australia. Mar Biol 105:73-82

Sebens KP (1994) Biodiversity of coral reefs: what are we losing and why? Am Zool 34:11.5-133

Sebens KP, Johnson AS (1991) Effects of water movement on prey capture and distribution of reef corals. Hydrobiologia 226:91-101

Smith SV (1981) The Houtman Abrolhos Islands: carbon metabolism of coral reefs at high latitude. Limnol Oceanogr 26 : $612-621$

Smith SV, Kımmerer WJ, Laws EA, Brock RC, Walsh TW (1981) Kanehoe Bay sewage diversion experiment: per- 
spectives on ecosystem responses to nutritional perturbation. Pacif Sci 35:279-395

Sorokin YI (1990a) Plankton in the reef ecosystems. In: Dubinsky Z (ed) Ecosystems of the world 25: coral reefs, Elsevier. Amsterdam, p 291-327

Sorokin YI (1990b) Aspects of trophic relations, productivity and energy balance in coral-reef ecosystems. In: Dubinsky $Z$ (ed) Ecosystems of the world 25: coral reefs. Elsevier, Amsterdam, p 401-410

Taniguchi A (1984) Microzooplankton biomass in the arctic and subarctic Pacific Ocean in summer. Mem Nat Inst Polar Res, Spec Issue 32:63-76

Ulanowicz RE (1984) Community measures of marine food networks and their possible applications. In: Fasham JR (ed) Flows of energy and materials in marine ecosystems. Plenum Press, New York, p 23-47

Valiela I (1984) Marine ecological processes. Springer-Verlag, New York, p 546

Wilkinson CR (1987) Microbial ecology on a coral reef. Search $18: 31-33$

This article was presented by D. M. Alongi (Senior Editorial Advisor), Townsville, Australia
Wilkinson CR (1993) Coral reefs of the world are facing widespread devastation: can we prevent this through sustainable management practices? Proc 7 th Int Symp Coral Reefs $1: 1-21$

Williams DMcB (1986) Temporal variation in the structure of reef slope fish communities (central Great Barier Reef): short term effects of Acanthaster plancl infestation. Mar Ecol Prog Ser 28:157-164

Williams DMcB, Hatcher Al (1983) Structure of fish communities on outer slopes of inshore, mid-shelf and outer shelf reefs of the Great Barrier Reef. Mar Ecol Prog Ser 10: $239-250$

Wulff F, Field JG, Mann KH (eds) (1989) Network analysis in marine ecology. Coastal and estuarine studies 32 . Springer Verlag, Berlin

Wulff F, Ulanowicz RE (1989) A comparative anatomy of the Baltic Sea and Chesapeake Bay ecosystems. In: Wulff $F_{\text {, }}$ Field JG, Mann $\mathrm{KH}$ (eds) Network analysis in marine ecology. Coastal and estuarine studies 32. Springer Verlag, Berlin

Manuscript first received: July 5, 1994

Revised version accepted: February 7, 1995 